\title{
Double Exchange in a Magnetically Frustrated System
}

\author{
R.S. Fishman \\ Condensed Matter Sciences Division, \\ Oak Ridge National Laboratory, Oak Ridge, TN 37831-6032
}

\begin{abstract}
This work examines the magnetic order and spin dynamics of a double-exchange model with competing ferromagnetic and antiferromagnetic Heisenberg interactions between the local moments. The Heisenberg interactions are periodically arranged in a Villain configuration in two dimensions with nearest-neighbor, ferromagnetic coupling $J$ and antiferromagnetic coupling $-\eta J$. This model is solved at zero temperature by performing a $1 / \sqrt{S}$ expansion in the rotated reference frame of each local moment. When $\eta$ exceeds a critical value, the ground state is a magnetically frustrated, canted antiferromagnet. With increasing hopping energy $t$ or magnetic field $B$, the local moments become aligned and the ferromagnetic phase is stabilized above critical values of $t$ or $B$. In the canted phase, a charge-density wave forms because the electrons prefer to sit on lines of sites that are coupled ferromagnetically. Due to a change in the topology of the Fermi surface from closed to open, phase separation occurs in a narrow range of parameters in the canted phase. In zero field, the long-wavelength spin waves are isotropic in the region of phase separation. Whereas the average spin-wave stiffness in the canted phase increases with $t$ or $\eta$, it exhibits a more complicated dependence on field. This work strongly suggests that the jump in the spin-wave stiffness observed in $\operatorname{Pr}_{1-x} \mathrm{Ca}_{x} \mathrm{MnO}_{3}$ with $0.3 \leq x \leq 0.4$ at a field of $3 \mathrm{~T}$ is caused by the delocalization of the electrons rather than by the alignment of the antiferromagnetic regions.
\end{abstract}




\section{INTRODUCTION}

The persistence of antiferromagnetic (AFM) short-range order in the ferromagnetic (FM) phase of the manganites has been recognized for many years [1]. In metallic manganites like $\mathrm{La}_{0.7} \mathrm{Ca}_{0.3} \mathrm{MnO}_{3}$ that contain a preponderance of AFM-coupled polaronic regions [2, 3], the Curie temperature $T_{\mathrm{C}}$ is suppressed but the magnetoresistance is strongly enhanced. Close to but below $T_{\mathrm{C}}$, the spin dynamics of $\mathrm{La}_{0.7} \mathrm{Ca}_{0.3} \mathrm{MnO}_{3}$ contains both a propagating spin-wave (SW) branch from the FM regions and a diffusive component from polaronic regions with suppressed FM interactions 4]. The low-temperature insulating phase of the manganite $\operatorname{Pr}_{1-x} \mathrm{Ca}_{x} \mathrm{MnO}_{3}$ with $0.3 \leq x \leq 0.4$ was originally believed [5, 6, 7] to be a canted AFM (CAF) but may actually contain regions with both FM and AFM short-range order 8, 9, 10, 11, 12, 13]. When an applied field $B$ exceeds about $3 \mathrm{~T}$, the resistivity drops by several orders of magnitude [6], the AFM regions shrink [12], and the SW stiffness $D_{\text {sw }}$ jumps by a factor of 3 [11]. Despite the central role played by AFM interactions in the manganites, little is known theoretically about how they affect the propagating SW dynamics. As first shown by Anderson and Hasegawa [14], the effective electron-hopping energy between two local moments making a relative angle $\Theta$ is proportional to $\cos \Theta / 2$ in the limit of large Hund's coupling. So AFM interactions may suppress the contribution of electron-mediated double-exchange (DE) to the SW dynamics [15]. By aligning the local moments, a magnetic field or electron hopping will alter the DE contribution to the SW dynamics. This paper examines the effects of AFM interactions on the ground-state properties and SW dynamics of electrons coupled to the local moments of a generalized Villain model [16, 17, 18, 19].

The generalized Villain model is one of the simplest periodic models to exhibit magnetic frustration. As described in Fig.1(a), the local moments $\mathbf{S}_{i}$ are coupled by the FM interaction $J$ along the $y$ direction and by either the FM interaction $J$ or the AFM interaction $-\eta J$ along the $x$ direction. The CAF phase is stable when $\eta$ exceeds the critical value $\eta_{c}$, which is $1 / 3$ when $\mathbf{B}=B \hat{\mathbf{z}}=0$ but increases as $B$ increases. Due to the different environments of the $a$ and $b$ sites, the angle $\theta_{b}$ at the $b$ sites is always larger than $\theta_{a}$ at the $a$ sites, as shown in Fig.1(b).

In the hybrid model considered here, the Heisenberg interactions between the local moments are in the Villain configuration while electrons with density $p=1-x$ are FM coupled to the local moments by Hund's coupling $J_{\mathrm{H}}$ and hop between neighboring sites with energy 
(a)

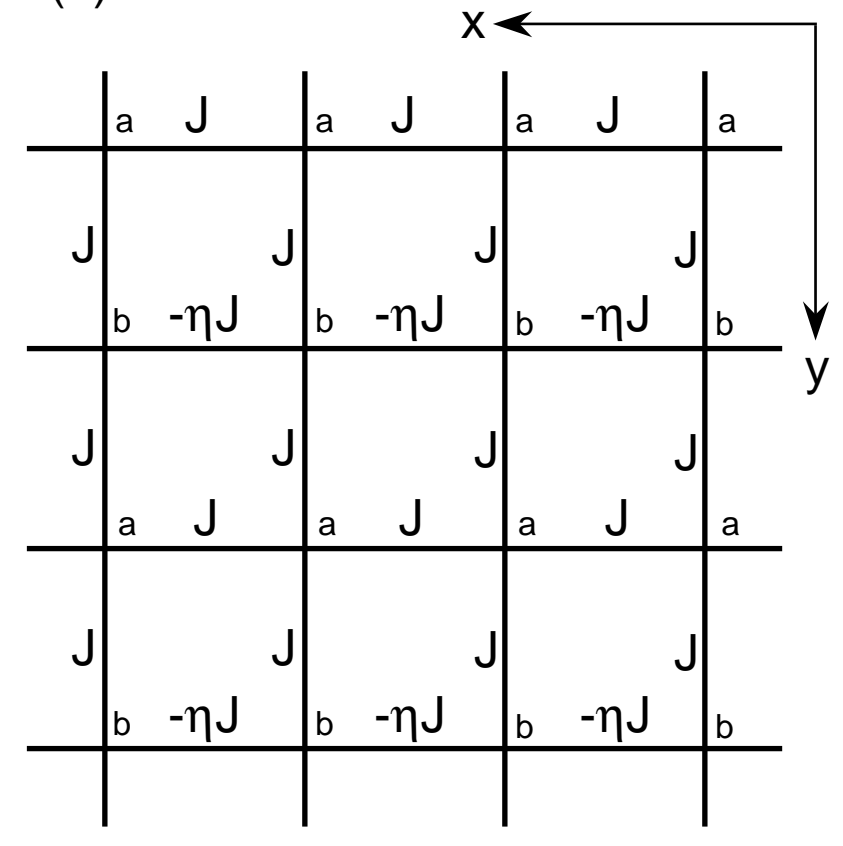

(b)

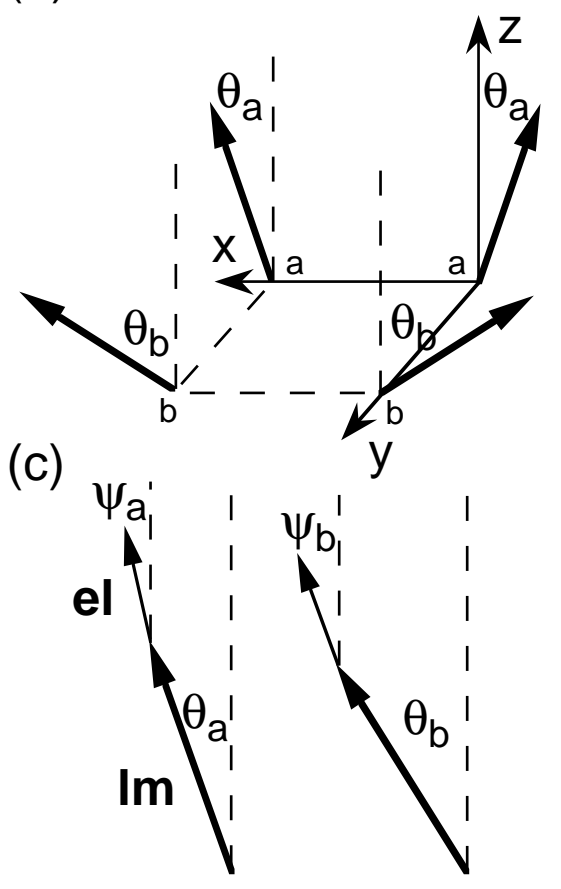

FIG. 1: (a) The generalized Villain model with Heisenberg couplings $J$ or $-\eta J$, (b) the local moments in the $x z$ plane subtend angles $\theta_{a}$ and $\theta_{b}$ with the $z$ axis, and (c) the electron spins also lie in the $x z$ plane but subtend angles $\psi_{a}<\theta_{a}$ and $\psi_{b}<\theta_{b}$ with the $z$ axis.

t. The DEV model (so called because it combines the DE and Villain models) provides several advantages as a basis for understanding the effects of AFM interactions and noncollinearity on the spin dynamics. First, except in a narrow range of parameters, a homogeneous CAF phase is stable against phase separation when the AFM control parameter $\eta$ exceeds $\eta_{c}$. By contrast, the well-studied hybrid model with AFM interactions $-J$ between all neighboring local moments phase separates before the AFM exchange $J$ is large enough to cant the spins 20, 21, 22, 23]. This phase instability is caused by a "site-local continuous degeneracy" 21, 23, 24] that is absent in the DEV model. Second, unlike the case in a hybrid model with AFM exchange only, the ground state of the DEV model contains a FM component even when $t=0$ and $B=0$. So it can be used to evaluate the change in SW stiffness $D_{\mathrm{sw}}$ as the electrons become mobile. Third, because the DEV model contains both FM and AFM Heisenberg interactions, it can be used to study insulating manganites like $\mathrm{Pr}_{0.66} \mathrm{Ca}_{0.34} \mathrm{MnO}_{3}$, where the AFM interactions are produced by superexchange and the FM interactions by short-ranged orbital order [25, 26]. 
For simplicity, we have constructed a model that is translationally symmetric in two dimensions. Since this model is solved at zero temperature, the qualitative results will be unchanged in three dimensions. More problematically, the AFM interactions are arranged periodically rather than in clusters. In the low-temperature phase of $\operatorname{Pr}_{0.67} \mathrm{Ca}_{0.33} \mathrm{MnO}_{3}$, the FM interactions may be confined to two-dimensional sheets with widths of roughly $25 \AA$ in a "red cabbage" structure [12, 13]. Neutron-scattering results [1]], on the other hand, suggest that the FM clusters in the insulating phase are about $40 \AA$ is diameter. So for wavelengths much longer than $40 \AA$, the SW's will average over the FM and AFM regions. Hence, the DEV model will provide qualitatively accurate predictions for the average SW stiffness $D_{\mathrm{sw}}^{a v}=\left(D_{\mathrm{sw}}^{x}+D_{\mathrm{sw}}^{y}\right) / 2$ defined in the long-wavelength limit.

The Hamiltonian of the DEV model is given by

$$
H=-t \sum_{\langle i, j\rangle} \sum_{\alpha}\left(c_{i \alpha}^{\dagger} c_{j \alpha}+c_{j \alpha}^{\dagger} c_{i \alpha}\right)-2 J_{\mathrm{H}} \sum_{i} \mathbf{s}_{i} \cdot \mathbf{S}_{i}-\sum_{\langle i, j\rangle} J_{i j} \mathbf{S}_{i} \cdot \mathbf{S}_{j}-B \sum_{i} S_{i z},
$$

where $c_{i \alpha}^{\dagger}$ and $c_{i \alpha}$ are the creation and destruction operators for an electron with spin $\alpha$ at site $i, \mathbf{s}_{i}=(1 / 2) c_{i \alpha}^{\dagger} \sigma_{\alpha \beta} c_{i \beta}$ is the electronic spin, and $\mathbf{S}_{i}$ is the spin of the local moment with magnitude $S$. Nearest-neighbor Heisenberg interactions $J_{i j}$ take the values $J$ (FM interaction) or $-\eta J$ (AFM interaction), as described in Fig.1(a). This model is solved at zero temperature by expanding the Hamiltonian in powers of $1 / \sqrt{S}$. To guarantee that the contributions to the SW frequencies from hopping and from the Heisenberg interactions are of the same order in $1 / \sqrt{S}, t$ is considered to be of the same order in $1 / \sqrt{S}$ as $J_{\mathrm{H}} S, J S^{2}$ and $B S$. Hence, the dimensionless parameters of our model are $t^{\prime}=t / J S^{2}, \eta, B^{\prime}=B / J S$, and $J_{\mathrm{H}} / J S$. To lowest order in $1 / S$, the magnetic field $B$ only couples to the local moments and not to the electrons. While the theory developed below can be extended to treat all values of the Hund's coupling, we shall for simplicity consider the limit of large $J_{\mathrm{H}} S$ or in dimensionless terms, $J_{\mathrm{H}} / J S \gg 1$ and $J_{\mathrm{H}} S / t \gg 1$.

This paper is divided into five sections. The Villain model is discussed in some detail in Section II, where we provide new results for the SW stiffness. The ground-state properties of the DEV model are presented in Section III. In Section IV, we evaluate the SW frequencies of the DEV model. Section V contains a discussion and summary. A short version of this work [27] is in press. 


\section{GENERALIZED VILLAIN MODEL}

This section presents, for the first time, a Holstein-Primakoff expansion for the generalized Villain model. In an equivalent approach, Saslow and Erwin [19] numerically evaluated the mode frequencies by linearizing the equations of motion for the spins. However, a formal Holstein-Primakoff expansion is required to lay the foundation for the solution of the full DEV model in Section IV.

The Hamiltonian of the generalized Villain model is given by Eq.(11) with $t=0$ and $J_{\mathrm{H}}=0$. The spin dynamics is immensely simplified in the rotated reference frame for each spin: $\overline{\mathbf{S}}_{i}=\underline{U}_{i}^{r o t} \mathbf{S}_{i}$, where $\underline{U}_{i}^{r o t}$ is the unitary rotation matrix for site $i$. A HolsteinPrimakoff expansion is performed within each rotated reference frame: $\bar{S}_{i z}=S-a_{i}^{\dagger} a_{i}$, $\bar{S}_{i x}+i \bar{S}_{i y}=\sqrt{2 S} a_{i}$, and $\bar{S}_{i x}-i \bar{S}_{i y}=\sqrt{2 S} a_{i}^{\dagger}$. Then the zeroth-order term (in powers of $1 / \sqrt{S})$ in $H_{v}$ can be written as

$$
E_{h}=\frac{1}{2} N J S^{2}\left\{-\cos 2 \theta_{a}+\eta \cos 2 \theta_{b}-2 \cos \left(\theta_{a}-\theta_{b}\right)-B^{\prime}\left(\cos \theta_{a}+\cos \theta_{b}\right)\right\}
$$

which is of order $J S^{2}$.

Minimizing $E_{h}$ with respect to $\theta_{a}$ and $\theta_{b}$ yields the relations

$$
\begin{gathered}
\sin 2 \theta_{a}+\sin \left(\theta_{a}-\theta_{b}\right)+\frac{1}{2} B^{\prime} \sin \theta_{a}=0, \\
-\eta \sin 2 \theta_{b}-\sin \left(\theta_{a}-\theta_{b}\right)+\frac{1}{2} B^{\prime} \sin \theta_{b}=0 .
\end{gathered}
$$

In zero field, it is easy to show that $\theta_{b}=3 \theta_{a}$ for all $\eta$. The equilibrium angles are plotted versus $\eta$ for several different values of $B^{\prime}$ ranging from 0 to 3 in the inset to Fig.2. In the limit of large $\eta$ with $B^{\prime}=0, \theta_{b} \rightarrow \pi / 2$ and $\theta_{a} \rightarrow \pi / 6$. For nonzero field, $\theta_{b}$ still approaches $\pi / 2$ but $\theta_{a}$ approaches an angle smaller than $\pi / 6$. In Fig.3(a), we plot the equilibrium angles versus $B^{\prime}$ for several values of $\eta$.

After linearizing Eqs.(3) and (44), we find that the phase boundary between the CAF and FM phases satisfies the relation

$$
B^{\prime}-2 \eta+4-2 \sqrt{(\eta+1)^{2}+1}=0
$$

which was first obtained by Gabay et al. [18]. While $\eta_{c}=1 / 3$ in zero field, $\eta_{c}$ increases with the field $B^{\prime}$ as seen in the inset to Fig.2 and in Fig.3(a). 


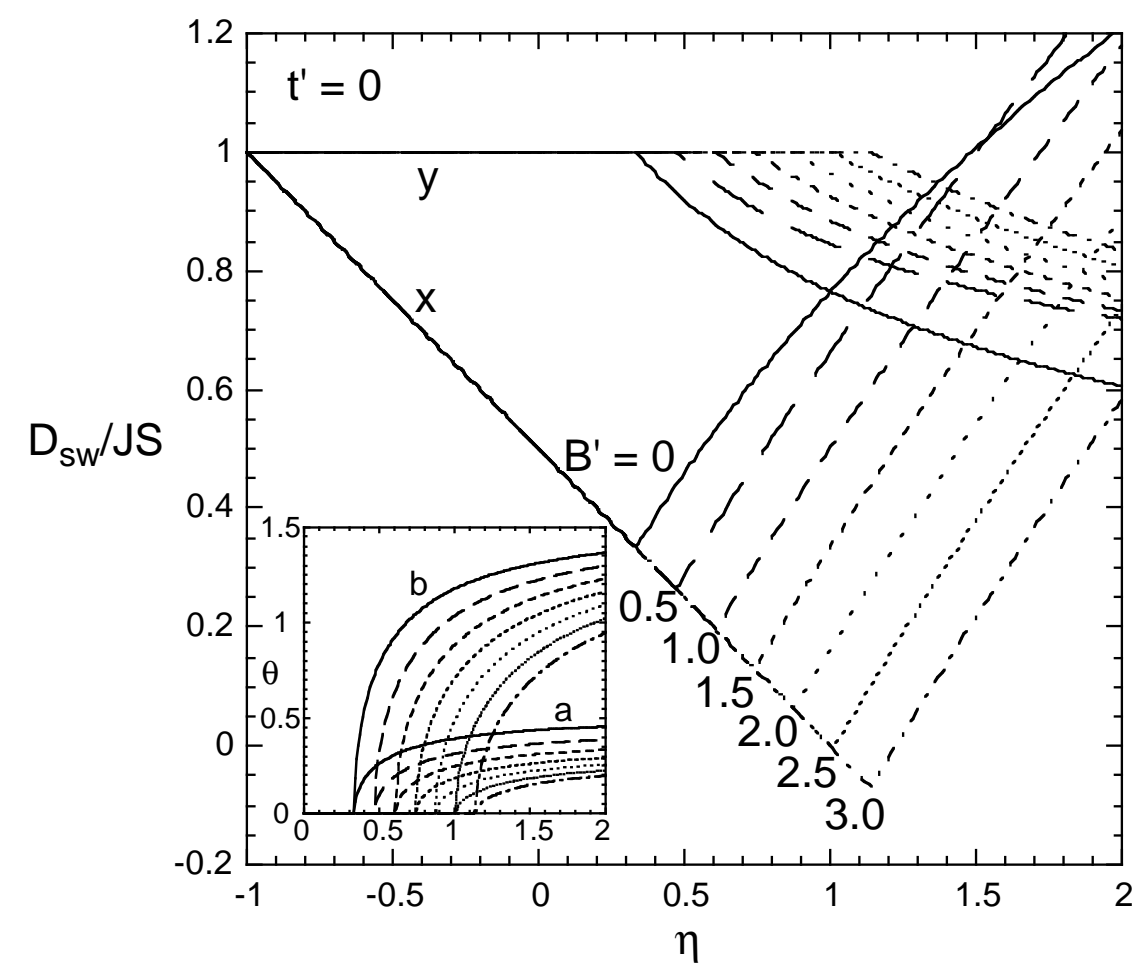

FIG. 2: The SW stiffnesses versus $\eta$ for $t^{\prime}=0$ and various values of the field $B^{\prime}$. In the inset are plotted the equilibrium angles $\theta_{a}$ and $\theta_{b}$ versus $\eta$ for the same set of fields.

Expanded as $H_{v}=E_{h}+H_{v 1}+H_{v 2}+\ldots$ in powers of $1 / \sqrt{S}$, the first-order term $H_{v 1}$ vanishes provided that the angles $\theta_{a}$ and $\theta_{b}$ satisfy Eqs.(3) and (44). In terms of the Fouriertransformed spin operators $a_{\mathbf{k}}^{(r)}$ and $a_{\mathbf{k}}^{(r) \dagger}$ on the $r=a$ or $b$ sublattice, the second-order term $H_{v 2}$ can be written as

$$
\begin{gathered}
H_{v 2}=J S \sum_{\mathbf{k}, r, s}\left\{a_{\mathbf{k}}^{(r) \dagger} a_{\mathbf{k}}^{(s)} A_{\mathbf{k}}^{(r, s)}+\left(a_{-\mathbf{k}}^{(r)} a_{\mathbf{k}}^{(s)}+a_{-\mathbf{k}}^{(r) \dagger} a_{\mathbf{k}}^{(s) \dagger}\right) B_{\mathbf{k}}^{(r, s)}\right\} \\
A_{\mathbf{k}}^{(a, a)}=2 \cos 2 \theta_{a}+2 \cos \left(\theta_{a}-\theta_{b}\right)-2 \cos ^{2} \theta_{a} \cos k_{x}+B^{\prime} \cos \theta_{a}, \\
A_{\mathbf{k}}^{(a, b)}=A_{\mathbf{q}}^{(b, a)}=-2 \cos ^{2}\left(\left(\theta_{a}-\theta_{b}\right) / 2\right) \cos k_{y}, \\
A_{\mathbf{k}}^{(b, b)}=2 \cos \left(\theta_{a}-\theta_{b}\right)-2 \eta \cos 2 \theta_{b}+2 \eta \cos ^{2} \theta_{b} \cos k_{x}+B^{\prime} \cos \theta_{b}, \\
B_{\mathbf{k}}^{(a, a)}=\sin ^{2} \theta_{a} \cos k_{x}, \\
B_{\mathbf{k}}^{(a, b)}=B_{\mathbf{k}}^{(b, a)}=\sin ^{2}\left(\left(\theta_{a}-\theta_{b}\right) / 2\right) \cos k_{y}, \\
B_{\mathbf{k}}^{(b, b)}=-\eta \sin ^{2} \theta_{b} \cos k_{x},
\end{gathered}
$$

where the lattice constant is set to one. 


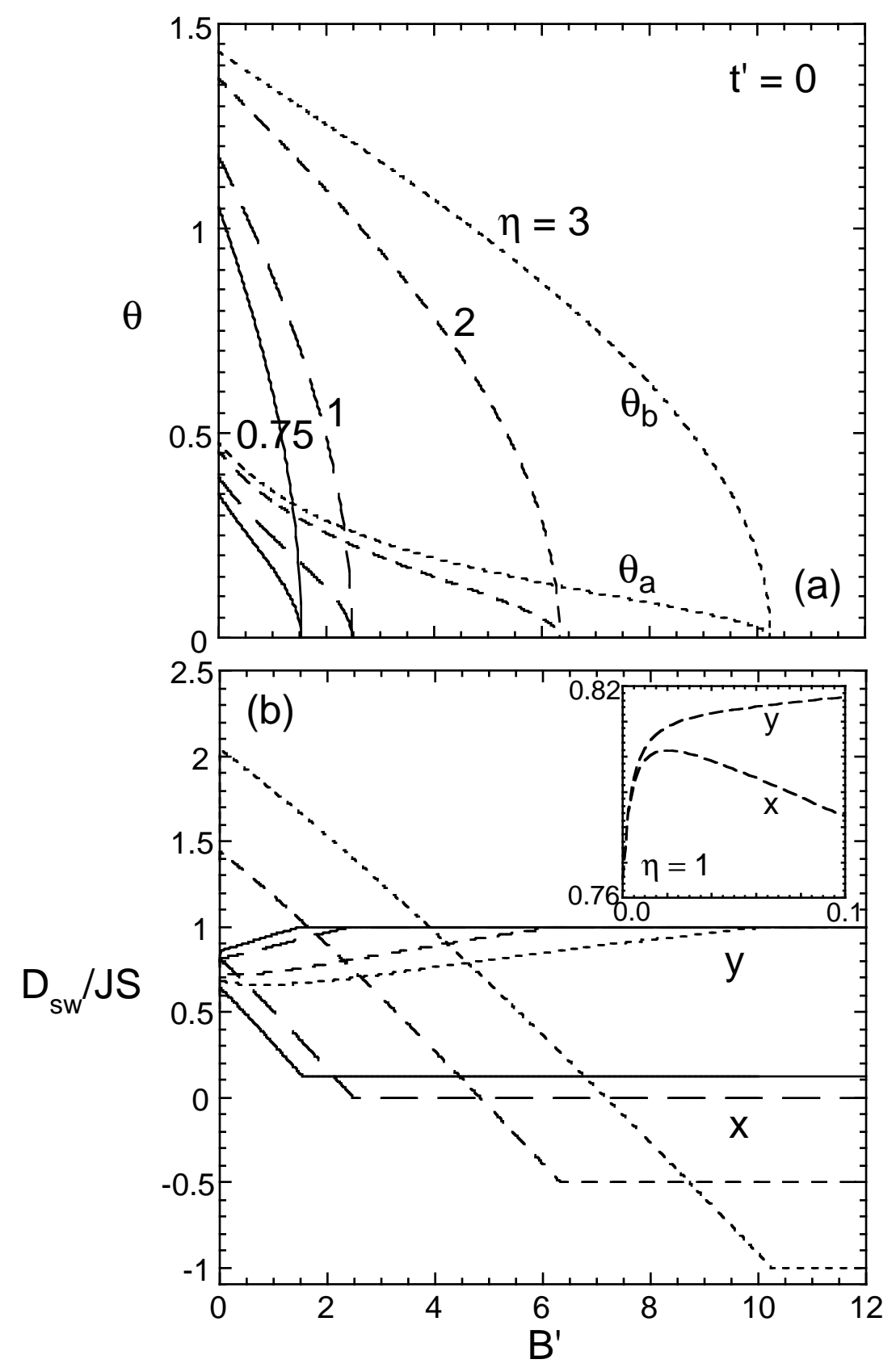

FIG. 3: (a) Equilibrium angles and (b) SW stiffnesses versus applied field $B^{\prime}$ for several values of $\eta$ and $t^{\prime}=0$. Inset in (b) are the SW stiffnesses when $\eta=1$ for very small fields and $k_{\alpha}=0.015 \pi$.

The Hamiltonian of Eq.(6) is easily diagonalized by applying the method of Walker and Walstedt [28], which was originally developed for spin glasses. The resulting spin excitation frequencies are given by

$$
\nu_{\mathbf{k}}^{ \pm}=\frac{J S}{\sqrt{2}} \sqrt{A_{\mathbf{k}}^{(a, a) 2}+A_{\mathbf{k}}^{(b, b) 2}+2\left(A_{\mathbf{k}}^{(a, b) 2}-B_{\mathbf{k}}^{(a, b) 2}\right)-4\left(B_{\mathbf{k}}^{(a, a) 2}+B_{\mathbf{k}}^{(b, b) 2}\right) \pm R_{\mathbf{k}}},
$$




$$
\begin{gathered}
R_{\mathbf{k}}^{2}=4\left\{A_{\mathrm{k}}^{(a, a) 2}+A_{\mathrm{k}}^{(b, b) 2}-4\left(B_{\mathbf{k}}^{(a, a) 2}+B_{\mathbf{k}}^{(b, b) 2}\right)\right\}\left(A_{\mathbf{k}}^{(a, b) 2}-B_{\mathbf{k}}^{(a, b) 2}\right) \\
+\left\{A_{\mathbf{k}}^{(a, a) 2}-A_{\mathbf{k}}^{(b, b) 2}-4\left(B_{\mathbf{k}}^{(a, a) 2}-B_{\mathbf{k}}^{(b, b) 2}\right)\right\}^{2}+8\left(A_{\mathbf{k}}^{(a, a)} A_{\mathbf{k}}^{(b, b)}+4 B_{\mathbf{k}}^{(a, a)} B_{\mathbf{k}}^{(b, b)}\right) \\
\times\left(A_{\mathbf{k}}^{(a, b) 2}+B_{\mathbf{k}}^{(a, b) 2}\right)-32 A_{\mathbf{k}}^{(a, b)} B_{\mathbf{k}}^{(a, b)}\left(A_{\mathbf{k}}^{(a, a)} B_{\mathbf{k}}^{(b, b)}+A_{\mathbf{k}}^{(b, b)} B_{\mathbf{k}}^{(a, a)}\right) .
\end{gathered}
$$

The SW frequency $\omega_{\mathbf{k}}=\nu_{\mathbf{k}}^{-}$satisfies the condition $\omega_{\mathbf{k}=0}=B$. Our results for $\nu_{\mathbf{k}}^{ \pm}$agree with the mode frequencies numerically evaluated by Saslow and Erwin [19]. In the longwavelength limit, the SW stiffnesses in the $x$ and $y$ directions are obtained from the expression $\lim _{\mathbf{k} \rightarrow 0} \omega_{\mathbf{k}}=B+D_{\mathrm{sw}}^{x} k_{x}^{2}+D_{\mathrm{sw}}^{y} k_{y}^{2}$.

In the FM phase, the SW frequency can be solved analytically:

$$
\omega_{\mathbf{k}}=B+J S\left(3-\eta+(\eta-1) \cos k_{x}\right)-J S \sqrt{(1+\eta)^{2}\left(1-\cos k_{x}\right)^{2}+4 \cos ^{2} k_{y}}
$$

So for $k_{x}=0, \omega_{\mathbf{k}}=B+2 J S\left(1-\cos k_{y}\right)$ is independent of $\eta$. The SW stiffnesses in the FM phase are given by the simple results $D_{\mathrm{sw}}^{x}=(J S / 2)(1-\eta)$ and $D_{\mathrm{sw}}^{y}=J S$, independent of field. The FM phase becomes unstable when $\omega_{\mathbf{Q}}=0$, where $\mathbf{Q}=(\pi, 0)$ is the AFM Bragg vector. This yields the same condition for the CAF-FM phase boundary as Eq.(51).

In the CAF phase, analytic results for the SW stiffnesses were found only when $B=0$ :

$$
\begin{gathered}
D_{\mathrm{sw}}^{x}=J S \sqrt{2} \eta \sqrt{1-\sqrt{\frac{\eta}{\eta+1}}}, \\
D_{\mathrm{sw}}^{y}=J S \sqrt{2} \sqrt{1-\sqrt{\frac{\eta}{\eta+1}}} .
\end{gathered}
$$

When $\eta \rightarrow \infty, D_{\mathrm{sw}}^{x} \rightarrow J S \sqrt{2 \eta}$ and $D_{\mathrm{sw}}^{y} \rightarrow J S \sqrt{2 / \eta}$. For $B>0, D_{\mathrm{sw}}^{y}$ tends to a nonzero limit but $D_{\mathrm{sw}}^{x}$ still diverges as $\eta \rightarrow \infty$. So the average SW stiffness always diverges when $\eta \rightarrow \infty$ regardless of the field.

As shown in Fig.2, $D_{\mathrm{sw}}^{x}$ is a linearly decreasing function of $\eta$ in the FM phase below $\eta_{c}$. In the CAF phase above $\eta_{c}, D_{\mathrm{sw}}^{x}$ increases and $D_{\mathrm{sw}}^{y}$ decreases with $\eta$. Long-wavelength SW's become isotropic when $D_{\mathrm{sw}}^{x}$ and $D_{\mathrm{sw}}^{y}$ cross. For $B=0, D_{\mathrm{sw}}^{x}=D_{\mathrm{sw}}^{y}$ when $\eta=1$. This crossing point moves to progressively larger values of $\eta$ with increasing field.

The SW stiffnesses are plotted versus field in Fig.3(b) for four different values of $\eta$. In the FM phase above $B_{c}$, the stiffnesses are independent of field. But in the CAF phase below $B_{c}$, the dependence on field is more complex. For $\eta>1, D_{\mathrm{sw}}^{x}>D_{\mathrm{sw}}^{y}$ at zero field and the stiffnesses cross as the field increases. If the SW stiffness in the $\alpha$ direction is defined 


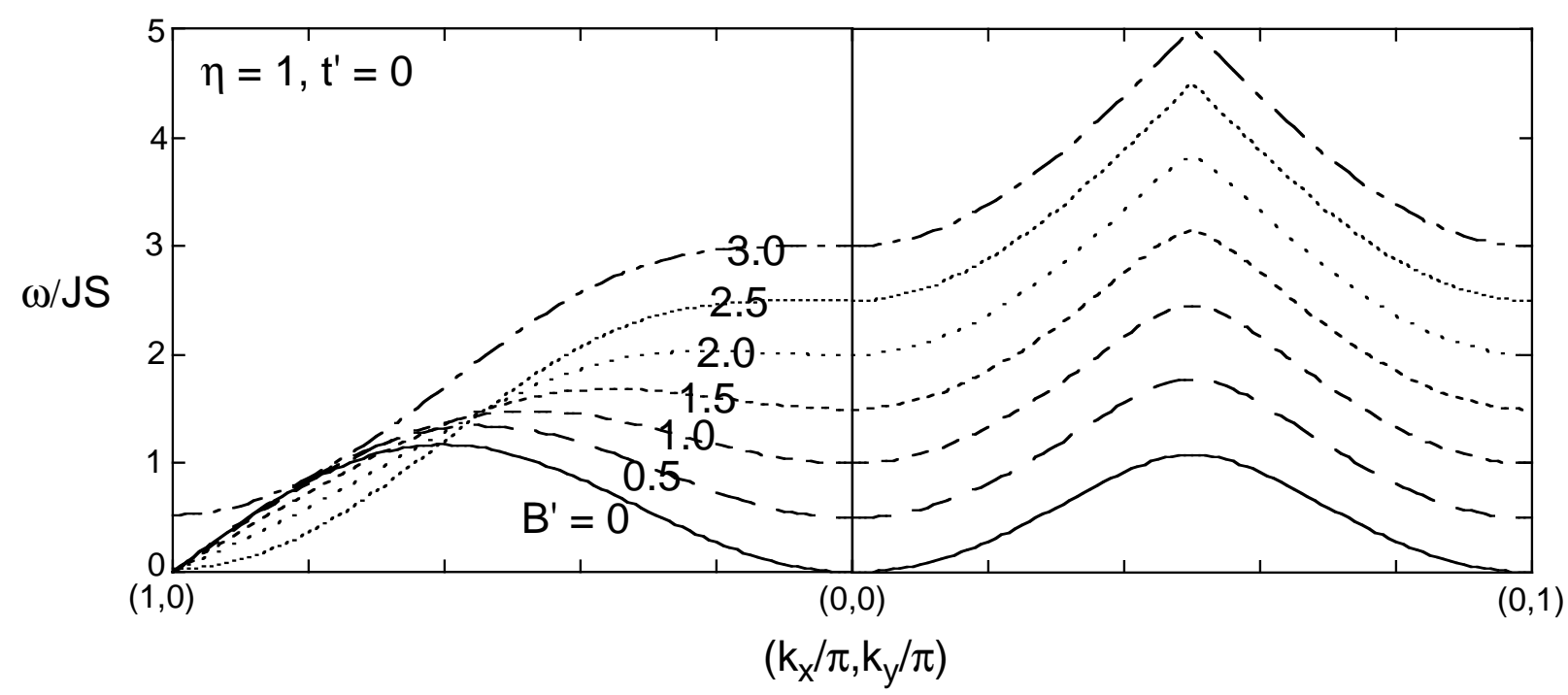

FIG. 4: The SW frequencies for $p=0.66, \eta=1, t^{\prime}=0$, and various fields $B^{\prime}$.

at fixed $k_{\alpha}$ by $D_{\mathrm{sw}}^{\alpha}\left(k_{\alpha}, B\right)=\left(\omega_{\mathrm{k}}-B\right) / k_{\alpha}^{2}$, then $D_{\mathrm{sw}}^{\alpha}\left(k_{\alpha}, B\right)$ increases rapidly near the field $B^{\star}=D_{\mathrm{sw}}^{\alpha}\left(k_{\alpha}, 0\right) k_{\alpha}^{2}$ as shown in the inset to Fig.3(b) for $k_{\alpha}=0.015 \pi$. As discussed in detail elsewhere 29], this behavior is typical of any CAF with a quadratic SW dispersion. Since the drop in $D_{\mathrm{sw}}^{x}$ is then steeper than the rise in $D_{\mathrm{sw}}^{y}, D_{\mathrm{sw}}^{a v}$ decreases with field for $B \gg B^{\star}$.

Surprisingly, Fig.3(b) indicates that the stiffness in the $x$ direction becomes negative for sufficiently large $\eta$. To understand this behavior, we have plotted the SW frequencies versus $\mathbf{k}$ for $\eta=1$ and several fields in Fig.4. Since $\omega_{\mathbf{Q}}=0$ and $\omega_{0}=B$ in the CAF phase, the SW stiffness in the $x$ direction must decrease as the field $B$ becomes comparable to $J S$. When $\eta=1, D_{\mathrm{sw}}^{x}=0$ for all fields above $B_{c}=2(\sqrt{5}-1) J S \approx 2.472 J S$. For $\eta>1$ and $B>B_{c}, D_{\mathrm{sw}}^{x}<0$ in the FM phase. Due to the reduced symmetry of the CAF phase, the first Brillouin zone extends from $-\pi$ to $\pi$ along $k_{x}$ but from $-\pi / 2$ to $\pi / 2$ along $k_{y}$ [30].

The SW frequencies are plotted versus $\mathbf{k}$ for $B=0$ and various values of $\eta$ in Fig.5. When $\eta=-1$, all of the Heisenberg interactions equal $J$ and the SW's are isotropic. For $\eta<\eta_{c}=1 / 3$ in the FM phase, the SW frequencies are independent of $\eta$ along $k_{y}$ but not along $k_{x}$, as implied by Eq.(15). With increasing $\eta$ in the CAF phase, the SW stiffness increases along the $x$ direction but decreases along the $y$ direction, as predicted by Eqs.(16) and (17) and shown in Fig.2. Also notice that the SW velocity at $\mathbf{Q}$ is an increasing function of $\eta$ in the CAF phase.

Both Figs.4 and 5 indicate that the SW velocity at $\mathbf{Q}$ softens as the CAF phase becomes 


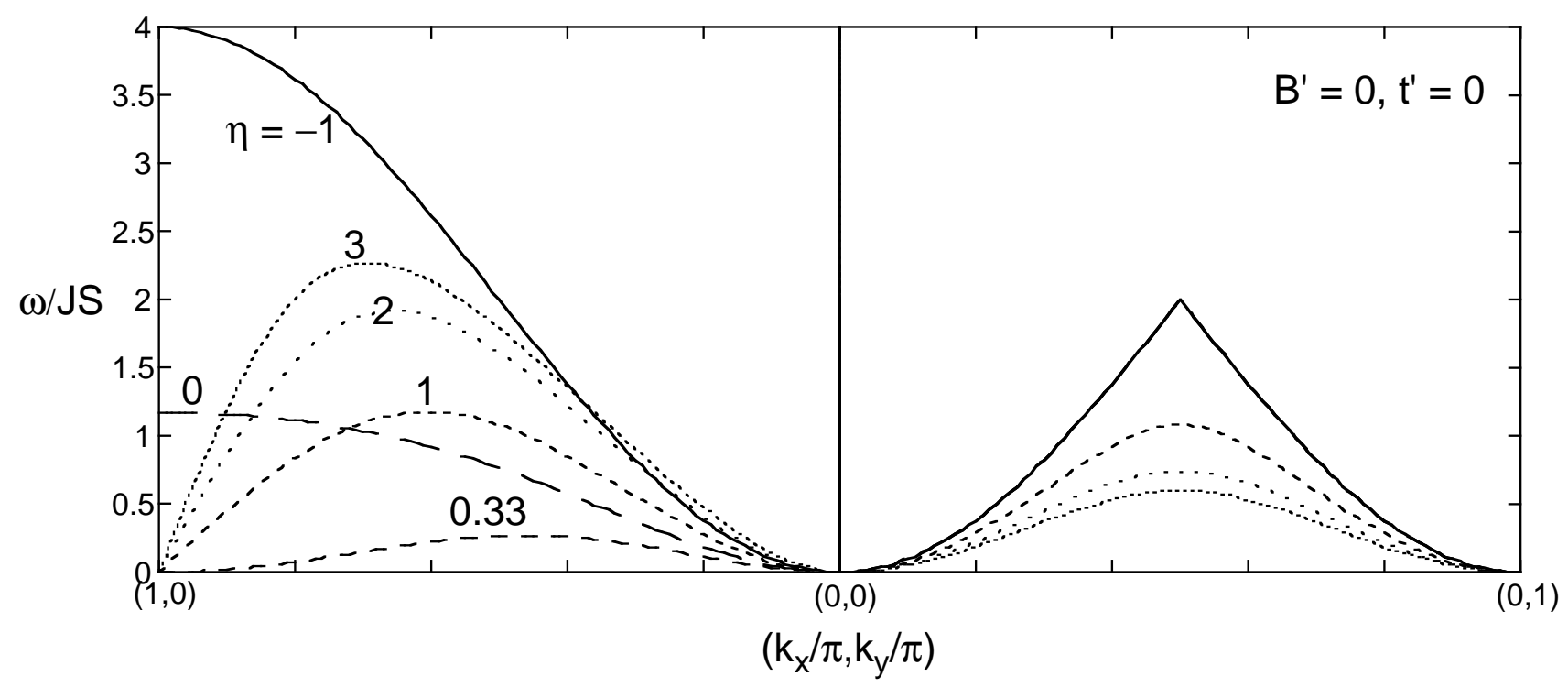

FIG. 5: The SW frequencies for $B^{\prime}=0, t^{\prime}=0$, and several values of $\eta$.

unstable. In the FM phase, $d \omega_{\mathbf{k}} / d k_{x}$ vanishes at $\mathbf{Q}$ for all $B>B_{c}$ or $\eta<\eta_{c}$. The softening of the SW velocity at the CAF-FM phase boundary is a quite general result discussed by Román and Soto [31].

\section{GROUND-STATE PROPERTIES OF THE DEV MODEL}

In this section, we discuss the ground-state properties of the DEV model, which is constructed by placing electrons with density $p$ on the Villain lattice. An electron on site $i$ is coupled to the local moment at that site by Hund's coupling $-2 J_{\mathrm{H}} \mathbf{s}_{i} \cdot \mathbf{S}_{i}$ and is allowed to hop to neighboring sites with hopping energy $t$, as specified by the DEV Hamiltonian of Eq.(11).

With the Fermion creation and destruction operators $\bar{c}_{\mathbf{k} \alpha}^{(r) \dagger}$ and $\bar{c}_{\mathbf{k} \alpha}^{(r)}$ defined in the reference frames of the local moments, the band Hamiltonian of the electrons can be written

$$
H_{b}=\sum_{\mathbf{k}} \sum_{i, j} \sum_{\alpha, \beta}\left(H_{b 0 \alpha \beta}^{i j}+V_{\alpha \beta}^{i j}(\mathbf{k})\right) \bar{c}_{\mathbf{k} \alpha}^{(i) \dagger} \bar{c}_{\mathbf{k} \beta}^{(j)},
$$

where $\bar{c}_{\mathbf{k} \alpha}^{(i)}=\left\{\bar{c}_{\mathbf{k} \alpha}^{(a)}, \bar{c}_{\mathbf{k}+\mathbf{Q}, \alpha}^{(a)}, \bar{c}_{\mathbf{k} \alpha}^{(b)}, \bar{c}_{\mathbf{k}+\mathbf{Q}, \alpha}^{(b)}\right\}$ defines the $i j$ subspace. The sum over $\mathbf{k}$ is restricted to the first Brillouin zone and $\alpha= \pm 1$ corresponds to spin up or down in the local reference frames. The zeroth-order band Hamiltonian includes just the Hund's coupling: $H_{b 0 \alpha \beta}^{i j}=$ 
$-J_{\mathrm{H}} S \delta_{i j} \sigma_{\alpha \beta}^{z}$. The potential $V_{\alpha \beta}^{i j}(\mathbf{k})$ is smaller by $t / J_{\mathrm{H}} S$ and has matrix elements

$$
\begin{gathered}
V_{\alpha \beta}^{11}(\mathbf{k})=-V_{\alpha \beta}^{22}(\mathbf{k})=-2 t \cos k_{x} \cos \theta_{a} \delta_{\alpha \beta}, \\
V_{\alpha \beta}^{33}(\mathbf{k})=-V_{\alpha \beta}^{44}(\mathbf{k})=-2 t \cos k_{x} \cos \theta_{b} \delta_{\alpha \beta}, \\
V_{\alpha \beta}^{12}(\mathbf{k})=-V_{\alpha \beta}^{21}(\mathbf{k})=-2 t i \cos k_{x} \sin \theta_{a} \sigma_{\alpha \beta}^{y}, \\
V_{\alpha \beta}^{34}(\mathbf{k})=-V_{\alpha \beta}^{43}(\mathbf{k})=-2 t i \cos k_{x} \sin \theta_{b} \sigma_{\alpha \beta}^{y} . \\
V_{\alpha \beta}^{13}(\mathbf{k})=V_{\alpha \beta}^{24}(\mathbf{k})=V_{\alpha \beta}^{31}(\mathbf{k})=V_{\alpha \beta}^{42}(\mathbf{k})=-2 t \cos k_{y} \cos \left(\left(\theta_{a}-\theta_{b}\right) / 2\right) \delta_{\alpha \beta}, \\
V_{\alpha \beta}^{14}(\mathbf{k})=V_{\alpha \beta}^{23}(\mathbf{k})=-V_{\alpha \beta}^{41}(\mathbf{k})=-V_{\alpha \beta}^{32}(\mathbf{k})=2 t i \cos k_{y} \sin \left(\left(\theta_{a}-\theta_{b}\right) / 2\right) \sigma_{\alpha \beta}^{y},
\end{gathered}
$$

Notice that the $\sigma_{\alpha \beta}^{y}$ terms couple the up and down spin states. Like $E_{h}$ of Eq.(2),$H_{b}$ is also of order $J S^{2}$.

The potential $V_{\alpha \beta}^{i j}(\mathbf{k})$ is treated within degenerate perturbation theory. Second-order perturbation theory with corrections of order $t / J_{\mathrm{H}} S$ will be required to obtain the $\mathrm{SW}$ frequencies in the next section. But to order $\left(t / J_{\mathrm{H}} S\right)^{0}$ or to first order in the potential, the spin up and down subspaces decouple and the band Hamiltonian $H_{b}$ is easily transformed into the diagonal form $H_{b}=\sum_{\mathbf{k}, \alpha, r} \epsilon_{\mathbf{k} \alpha}^{(r)} d_{\mathbf{k} \alpha}^{(r) \dagger} d_{\mathbf{k} \alpha}^{(r)}$ by the rotations $\bar{c}_{\mathbf{k} \alpha}^{(a)}=u_{\mathbf{k}}^{(a)} d_{\mathbf{k} \alpha}^{(a)}+u_{\mathbf{k}}^{(b)} d_{\mathbf{k} \alpha}^{(b)}$ and $\bar{c}_{\mathbf{k} \alpha}^{(b)}=u_{\mathbf{k}}^{(b)} d_{\mathbf{k} \alpha}^{(a)}-u_{\mathbf{k}}^{(a)} d_{\mathbf{k} \alpha}^{(b)}$ where $\epsilon_{\mathbf{k} \alpha}^{(r)}=-J_{\mathrm{H}} S \alpha+\tilde{\epsilon}_{\mathbf{k}}^{(r)}$,

$$
\begin{gathered}
u_{\mathbf{k}}^{(a) 2}=1-u_{\mathbf{k}}^{(b) 2}=\frac{1}{2}\left\{1+\frac{1}{w_{\mathbf{k}}}\left(\cos \theta_{a}-\cos \theta_{b}\right) \cos k_{x}\right\}, \\
w_{\mathbf{k}}=\sqrt{\left(\cos \theta_{a}-\cos \theta_{b}\right)^{2} \cos ^{2} k_{x}+4 \cos ^{2}\left(\left(\theta_{a}-\theta_{b}\right) / 2\right) \cos ^{2} k_{y}} .
\end{gathered}
$$

The upper and lower signs in $\tilde{\epsilon}_{\mathbf{k}}^{(r)}$ refer to the $r=a$ and $b$ bands, respectively. With $H_{b}$ in diagonal form, $E_{b}=\left\langle H_{b}\right\rangle$ is easy to evaluate.

It is straightforward to minimize the zeroth-order energy $E_{0}=E_{h}+E_{b}$ with respect to the angles $\theta_{a}$ and $\theta_{b}$ in the limit of large $J_{\mathrm{H}} S$. The relations that generalize Eqs.(3) and (44) are

$$
\begin{gathered}
\sin 2 \theta_{a}+\sin \left(\theta_{a}-\theta_{b}\right)+\frac{1}{2} B^{\prime} \sin \theta_{a}+\frac{1}{N J S^{2}} \sum_{\mathbf{k}}\left\{\frac{d \tilde{\epsilon}_{\mathbf{k}}^{(a)}}{d \theta_{a}} f\left(\tilde{\epsilon}_{\mathbf{k}}^{(a)}\right)+\frac{d \tilde{\epsilon}_{\mathbf{k}}^{(b)}}{d \theta_{a}} f\left(\tilde{\epsilon}_{\mathbf{k}}^{(b)}\right)\right\}=0, \\
-\eta \sin 2 \theta_{b}-\sin \left(\theta_{a}-\theta_{b}\right)+\frac{1}{2} B^{\prime} \sin \theta_{b}+\frac{1}{N J S^{2}} \sum_{\mathbf{k}}\left\{\frac{d \tilde{\epsilon}_{\mathbf{k}}^{(a)}}{d \theta_{b}} f\left(\tilde{\epsilon}_{\mathbf{k}}^{(a)}\right)+\frac{d \tilde{\epsilon}_{\mathbf{k}}^{(b)}}{d \theta_{b}} f\left(\tilde{\epsilon}_{\mathbf{k}}^{(b)}\right)\right\}=0,
\end{gathered}
$$


where $f(\tilde{\epsilon})=\Theta(\tilde{\mu}-\tilde{\epsilon})$ is the Fermi function at $T=0$ and $\tilde{\mu}=\mu-J_{\mathrm{H}} S \operatorname{sgn}(p-1)$ is the shifted chemical potential. For a fixed $\eta$ and $B^{\prime}$, the equilibrium angles decrease with increasing $t^{\prime}$. The phase boundary between the CAF and FM phases is now given by the condition

$$
B^{\prime}-2 \eta+4+3 K / 4 J S^{2}-2 \sqrt{(1+\eta)^{2}+1+K / 4 J S^{2}+\left(K / 8 J S^{2}\right)^{2}}=0,
$$

where $K=-\left(\left\langle\tilde{\epsilon}_{\mathbf{k}}^{(a)}\right\rangle+\left\langle\tilde{\epsilon}_{\mathbf{k}}^{(b)}\right\rangle\right) / 2 \geq 0$ is the average kinetic energy of the electrons in the FM phase. This reduces to Eq.(5) when $K=0$. For $p=0.66, \eta=2$, and $B^{\prime}=0$, the dependence of the equilibrium angles on $t^{\prime}$ is plotted in Fig.6(a). Also shown is the average spin $M=S\left(\cos \theta_{a}+\cos \theta_{b}\right) / 2$ of the local moments. When $t^{\prime}=10$ and $B^{\prime}=0$, the equilibrium angles and $M / S$ are plotted versus $\eta$ in Fig.7(a). Their dependence on field $B^{\prime}$ is plotted in Fig.8(a) for $t^{\prime}=3$ and $\eta=2$.

Surprisingly, the electronic occupation of the $a$ and $b$ sites on the Villain lattice are different. For $p<1$, the occupancies of the $a$ and $b$ sublattices are given by

$$
\begin{aligned}
& n_{a}=\frac{2}{N} \sum_{\mathbf{k}}\left\{u_{\mathbf{k}}^{(a) 2} f\left(\tilde{\epsilon}_{\mathbf{k}}^{(a)}\right)+u_{\mathbf{k}}^{(b) 2} f\left(\tilde{\epsilon}_{\mathbf{k}}^{(b)}\right)\right\}, \\
& n_{b}=\frac{2}{N} \sum_{\mathbf{k}}\left\{u_{\mathbf{k}}^{(b) 2} f\left(\tilde{\epsilon}_{\mathbf{k}}^{(a)}\right)+u_{\mathbf{k}}^{(b) 2} f\left(\tilde{\epsilon}_{\mathbf{k}}^{(a)}\right)\right\},
\end{aligned}
$$

From the relation $u_{\mathbf{k}}^{(a) 2}+u_{\mathbf{k}}^{(b) 2}=1$, it follows that $\left(n_{a}+n_{b}\right) / 2=p$ is just the average number of electrons per site.

In the CAF phase, electrons prefer to sit on the $a$ sites of the Villain lattice. The fraction $f_{a}=n_{a} / 2 p \geq 1 / 2$ of such electrons is plotted in Figs.(6-8). For $\eta=2$ and $B=0$ in Fig.6(a), $f_{a}$ has a maximum of 0.574 as $t^{\prime} \rightarrow 0$ and approaches $1 / 2$ as $t^{\prime} \rightarrow t_{c}^{\prime} \approx 13.2$. Similar behavior is found in Figs.7(a) and 8(a), where $f_{a}$ is shown to be an increasing function of $\eta$ and a decreasing function of $B^{\prime}$. This behavior is easy to understand: the largest angles between neighboring spins are along the $x$ axis between $b$ sites with angles differing by $2 \theta_{b}$. When an electron hops onto a $b$ site, it cannot easily hop to other $b$ sites and so quickly moves onto a neighboring $a$ site, where it can readily travel between other $a$ sites with angular difference $2 \theta_{a} \ll 2 \theta_{b}$. Hence, the non-collinearity of the local moments quite naturally produces a charge-density wave (CDW) with a substantial amplitude. As $\eta \rightarrow \infty, \theta_{b}$ approaches $\pi / 2$ and $f_{a}$ approaches 0.593 . So even when the electrons are unable to hop between sites on the $b$ sublattice (since the angles on neighboring $b$ sites differ by $\pi$ ), roughly $40 \%$ of the 

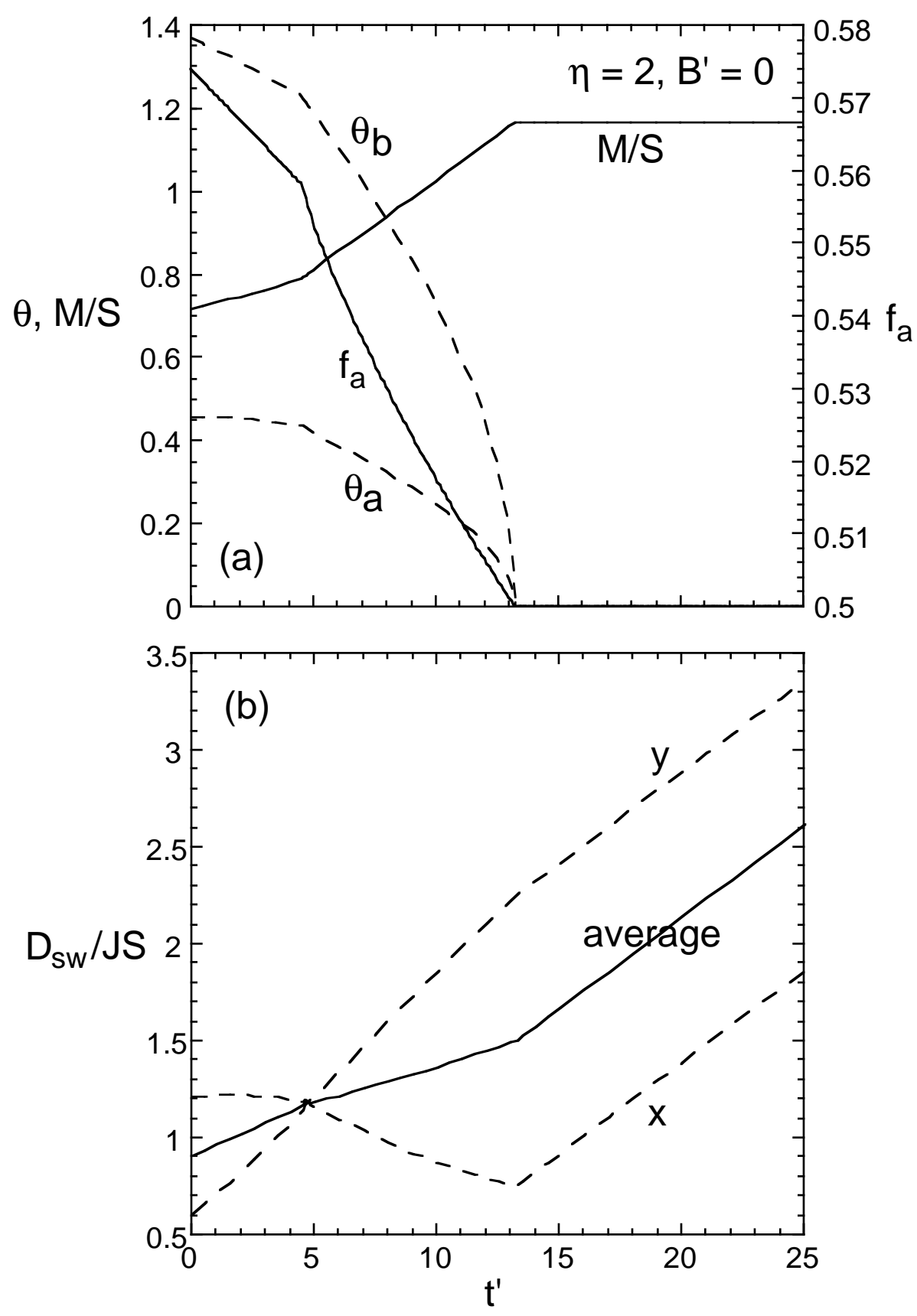

FIG. 6: (a) The angles $\theta_{a}$ and $\theta_{b}$ of the local moments, the total local magnetization $M / S$, the fraction $f_{a}$ of the electrons on the $a$ sites, and (b) the spin-wave stiffnesses (both in the $x$ and $y$ directions and their average) for $p=0.66, \eta=2$, and $B^{\prime}=0$ versus $t^{\prime}$.

electrons can still be found on $b$ sites at any one time. Because neither the CAF nor FM densities-of-states contain a gap, both phases are metallic within the DEV model for $t^{\prime}>0$. Due to short-range orbital ordering, a CDW with the same period as the one predicted 

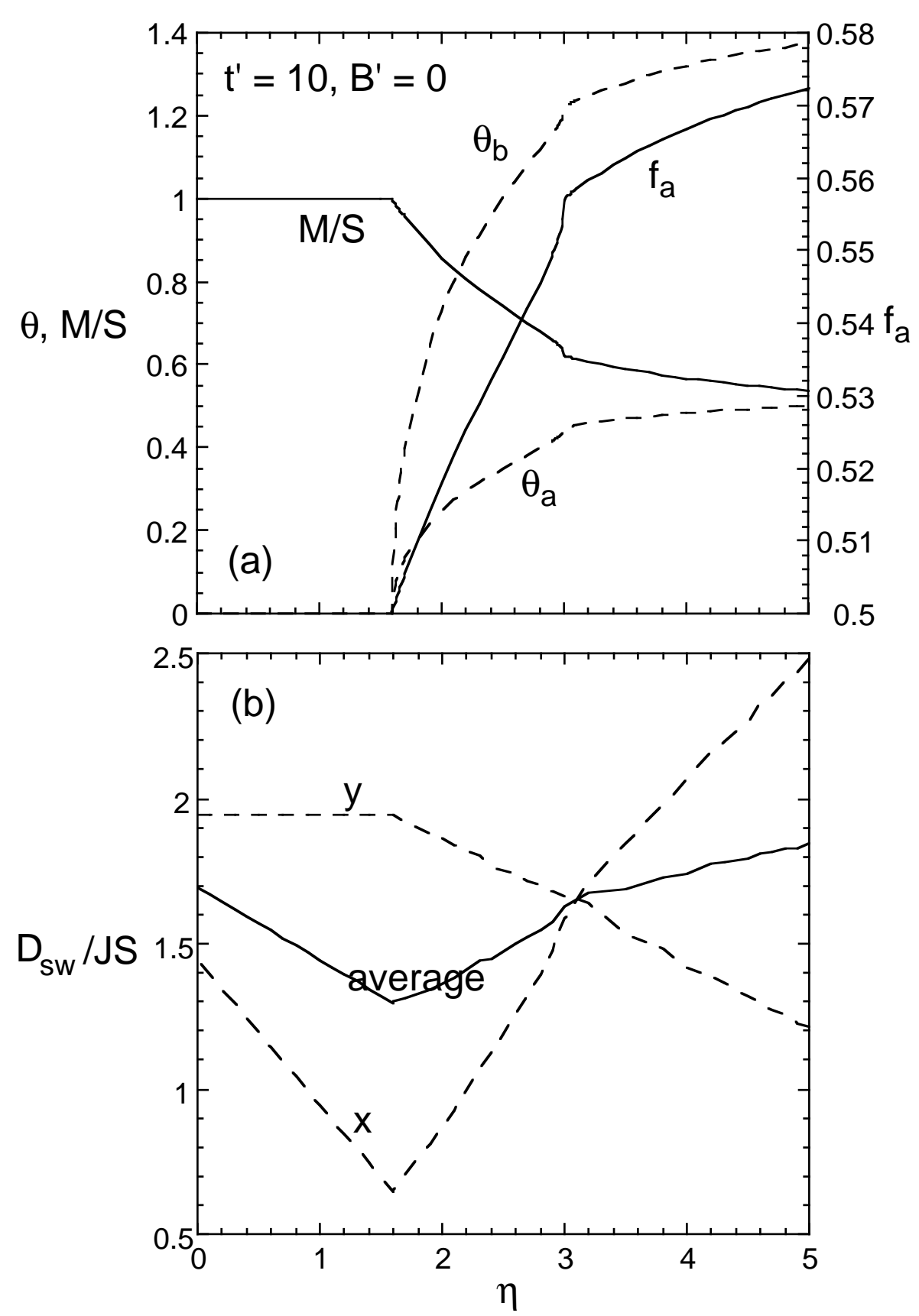

FIG. 7: (a) The angles $\theta_{a}$ and $\theta_{b}$ of the local moments, the total local magnetization $M / S$, the fraction $f_{a}$ of the electrons on the $a$ sites, and (b) the spin-wave stiffnesses for $p=0.66, t^{\prime}=10$, and $B^{\prime}=0$ versus $\eta$.

here has in fact been observed in the AFM regions of $\operatorname{Pr}_{0.7} \mathrm{Ca}_{0.3} \mathrm{MnO}_{3}$ [7, 32]. However, the observed charge ordering is perfect: all of the $\mathrm{Mn}^{3+}$ ions lie on one sublattice and all of the $\mathrm{Mn}^{4+}$ ions lie on the other. Such perfect charge ordering is never achieved within the DEV model. 

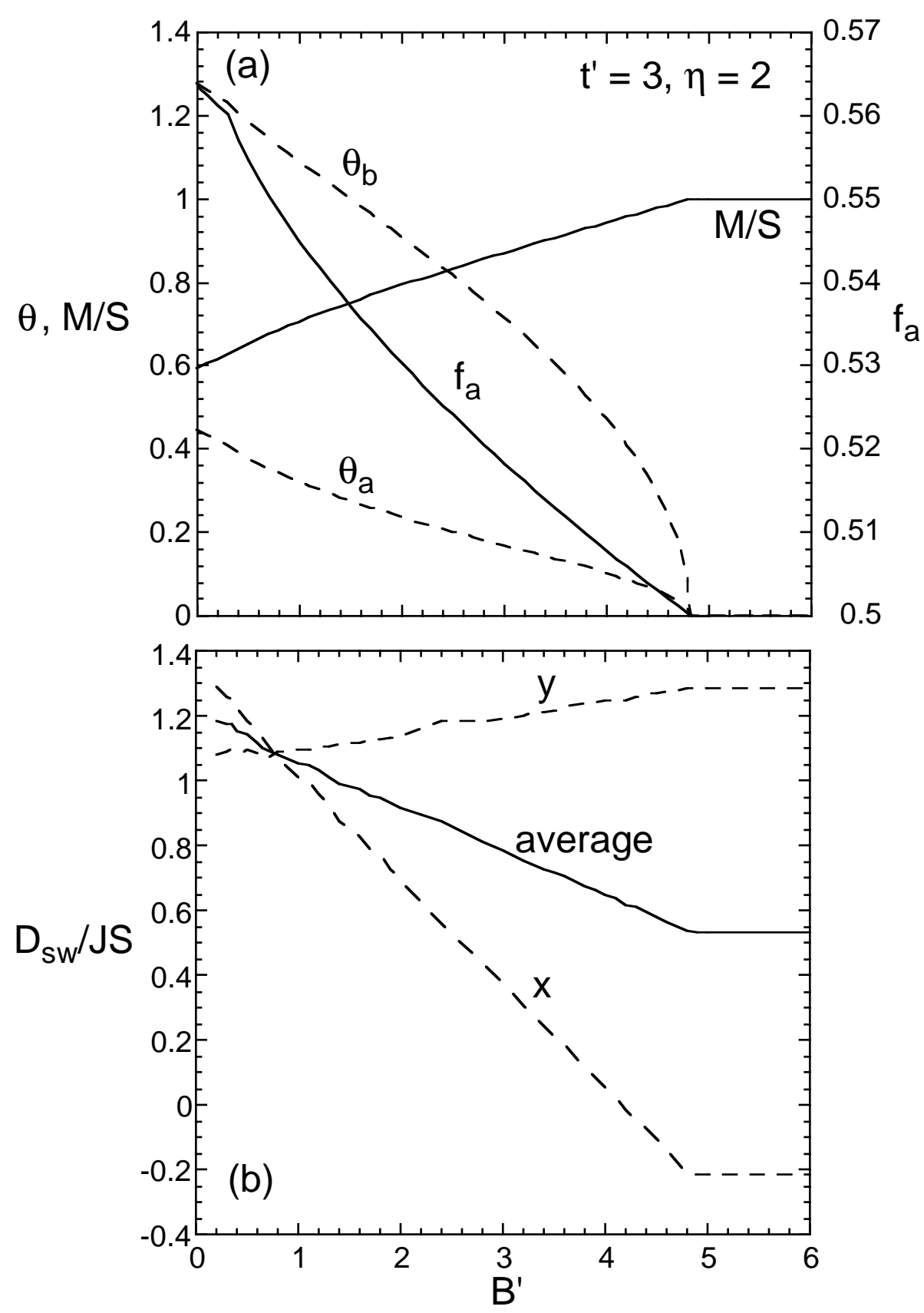

FIG. 8: (a) The angles $\theta_{a}$ and $\theta_{b}$ of the local moments, the total local magnetization $M / S$, the fraction $f_{a}$ of the electrons on the $a$ sites, and (b) the spin-wave stiffnesses for $p=0.66, t^{\prime}=3$, and $\eta=2$ versus $B^{\prime}$.

Another surprising result is that phase separation occurs within a narrow range of $t^{\prime}$. In a plot of filling $p$ versus chemical potential $\mu$, phase separation appears as a jump $\Delta p$ in $p(\mu)$. For the parameters $\eta=3$ and $B=0$ in Fig.9, $\Delta p$ reaches a maximum of about 0.0028 when $t^{\prime} \approx 10.0$ and shrinks as $t^{\prime}$ increases. If $p$ is fixed at 0.66 , then phase separation 


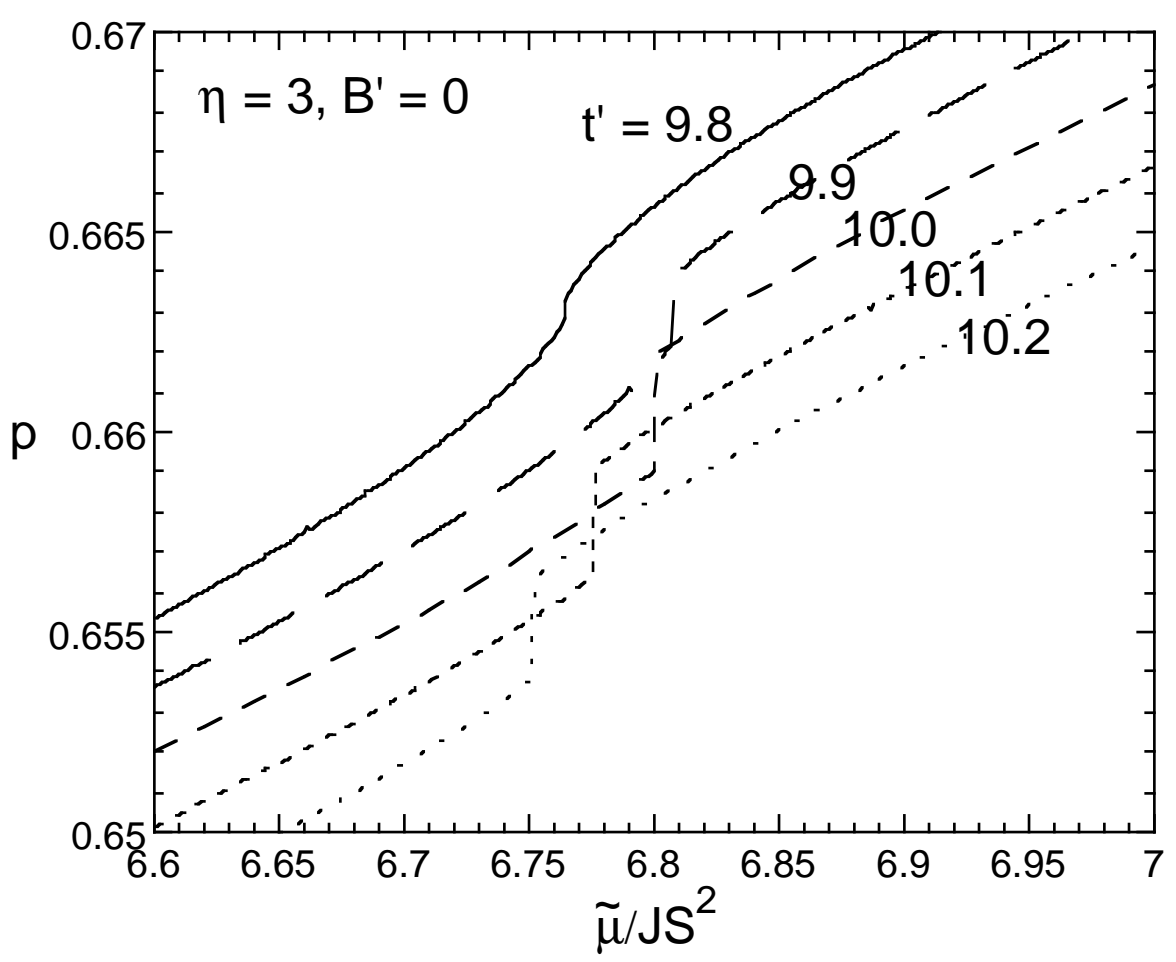

FIG. 9: The filling $p$ versus chemical potential $\tilde{\mu}$ for various values of $t^{\prime}, \eta=3$, and $B^{\prime}=0$. Phase separation appears as discontinuities in $p$.

occurs within a very narrow range of $t^{\prime}$ between about 9.98 and 10.02. For fixed $p$, phase separation appears as jumps in the equilibrium angles $\theta_{r}$ and in the electron fraction $f_{a}$, as seen in Figs.6(a) and 7(a) for zero field.

Like the Pomeranchuk instability [33, 34] in the two-dimensional Hubbard model, the phase instability in the DEV model occurs close to but slightly above the Van Hove filling and is marked by a change in Fermi surface (FS) topology from closed to open. In the usual Pomeranchuk instability, however, the change in FS topology spontaneously breaks the square symmetry of the lattice. Square symmmetry is already broken in the DEV model by the Villain arrangement of the Heisenberg interactions. The FS of the DEV model is sketched in Fig.10 for $\eta=3, B=0, p=0.66$, and for values of $t^{\prime}$ on either side of the phase-separated range. For $t^{\prime}$ just above 10.0, the extra electrons in the neck of the $a$ FS around $\mathbf{k}=0$ are offset by the holes in the $b$ FS around $\mathbf{k}=(\pi, \pi / 2)$. Although phase separation occurs for any $t^{\prime}$ around some value of the filling, it becomes significant only if the $b$ FS is already present when the necks in the $a$ FS develop. For the parameters in Fig.9, this requires that $t^{\prime} \geq 9.9$. 


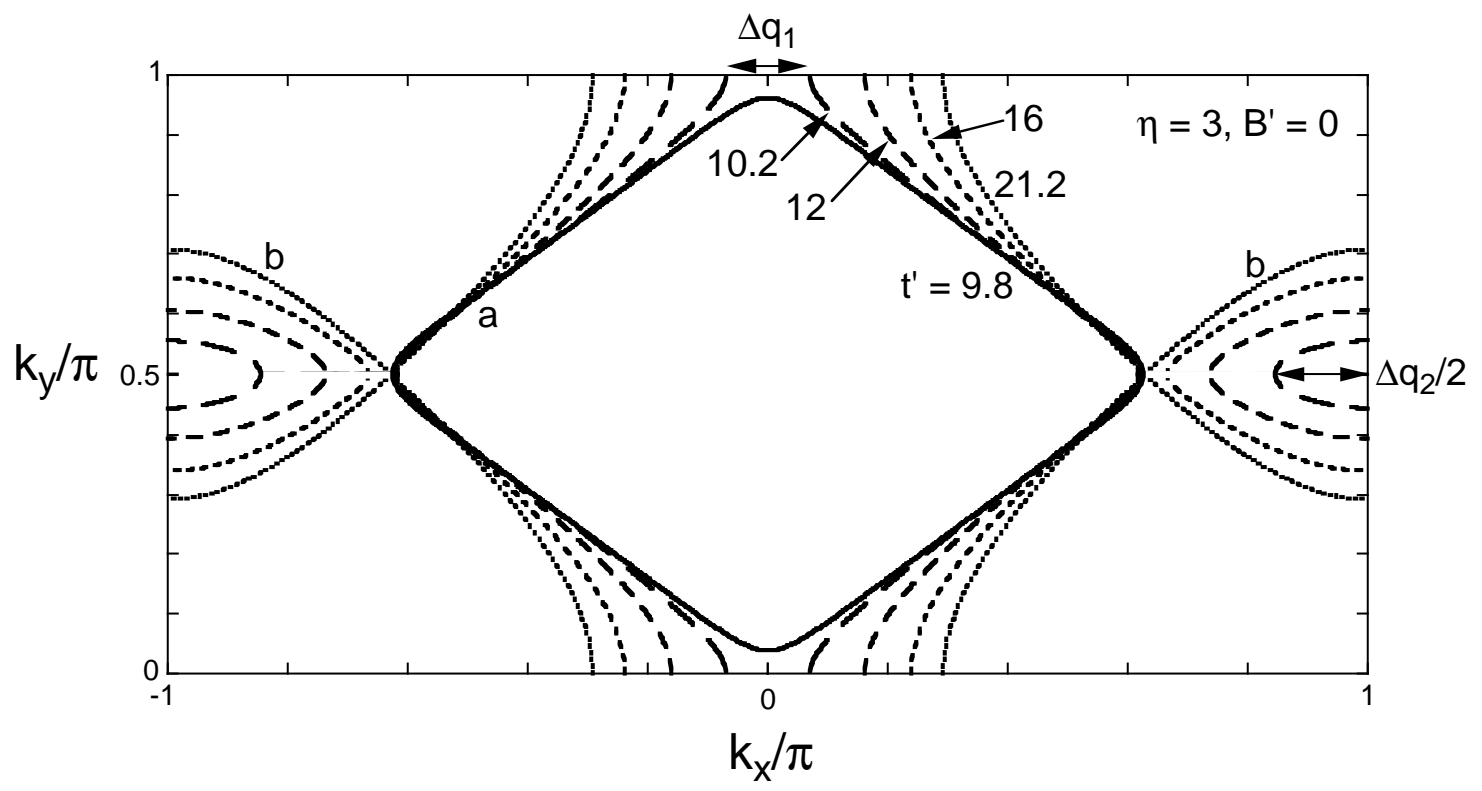

FIG. 10: The FS is plotted for $\eta=3, B^{\prime}=0, p=0.66$, and $t^{\prime}=9.8,10.2,12,16$, or 21.2. For $t^{\prime}>10$, the $a$ FS develops necks around $\mathbf{k}=0$.

A magnetic field very quickly narrows and then eliminates the region of phase separation. For $t^{\prime}=3$ and $\eta=2$, phase separation does not occur with increasing field. The small kinks in the equilibrium angles and electron fraction seen in Fig.8(a) correspond to points of Van Hove filling (where the necks in the $a$ FS first appear) but not to a phase-separated region.

\section{SPIN DYNAMICS OF THE DEV MODEL}

In this section, we evaluate the SW frequencies for the DEV model by following the general formalism developed by Golosov [23]. While introducing some new physics, the presence of non-collinear spins also complicates things a bit.

When $J_{\mathrm{H}} S / t$ is finite, the relationships given above for $\bar{c}_{\mathbf{k} \alpha}^{(r)}$ in terms of $d_{\mathbf{k} \alpha}^{(s)}$ must contain admixtures of the opposite-spin terms $\left(t / J_{\mathrm{H}} S\right) d_{\mathbf{k}+\mathbf{Q},-\alpha}^{(s)}$. As sketched in Fig.1(c), this implies that the equilibrium angles $\psi_{r}$ of the electrons are smaller than the angles $\theta_{r}$ of the local moments because the electrons try to align their spins as much as possible. For large Hund's coupling, $\theta_{r}-\psi_{r} \propto t / J_{\mathrm{H}} S$ and the electrons always exert a small torque on the local moments. In the local reference frame of site $i$ on the $r$ sublattice, $\left\langle\bar{s}_{i x}\right\rangle=\left(n_{r} / 2\right) \sin \left(\theta_{r}-\psi_{r}\right)$ so these new terms produce a correction to the Hund's coupling $-J_{\mathrm{H}} \mathbf{S}_{i} \cdot \mathbf{s}_{i}$ that survives in 
the $J_{\mathrm{H}} S \rightarrow \infty$ limit. Indeed, these torque terms are required to obtain sensible results for the SW frequencies.

To second order in perturbation theory, the new relationships for the Fermion operators are given by

$$
\begin{aligned}
\bar{c}_{\mathbf{k} \alpha}^{(a)}=u_{\mathbf{k}}^{(a)} d_{\mathbf{k} \alpha}^{(a)}+ & u_{\mathbf{k}}^{(b)} d_{\mathbf{k} \alpha}^{(b)}+\frac{t}{J_{\mathrm{H}} S}\left\{\left(u_{\mathbf{k}}^{(a)} \cos k_{y} \sin \left(\left(\theta_{a}-\theta_{b}\right) / 2\right)-u_{\mathbf{k}}^{(b)} \cos k_{x} \sin \theta_{a}\right) d_{\mathbf{k}+\mathbf{Q},-\alpha}^{(a)}\right. \\
& \left.-\left(u_{\mathbf{k}}^{(a)} \cos k_{x} \sin \theta_{a}+u_{\mathbf{k}}^{(b)} \cos k_{y} \sin \left(\left(\theta_{a}-\theta_{b}\right) / 2\right)\right) d_{\mathbf{k}+\mathbf{Q},-\alpha}^{(b)}\right\} \\
\bar{c}_{\mathbf{k} \alpha}^{(b)}=u_{\mathbf{k}}^{(b)} d_{\mathbf{k} \alpha}^{(a)}- & u_{\mathbf{k}}^{(a)} d_{\mathbf{k} \alpha}^{(b)}+\frac{t}{J_{\mathrm{H}} S}\left\{-\left(u_{\mathbf{k}}^{(a)} \cos k_{x} \sin \theta_{b}+u_{\mathbf{k}}^{(b)} \cos k_{y} \sin \left(\left(\theta_{a}-\theta_{b}\right) / 2\right)\right) d_{\mathbf{k}+\mathbf{Q},-\alpha}^{(a)}\right. \\
& \left.+\left(-u_{\mathbf{k}}^{(a)} \cos k_{y} \sin \left(\left(\theta_{a}-\theta_{b}\right) / 2\right)+u_{\mathbf{k}}^{(b)} \cos k_{x} \sin \theta_{b}\right) d_{\mathbf{k}+\mathbf{Q},-\alpha}^{(b)}\right\}
\end{aligned}
$$

On the $r$ sublattice, these equations imply that $\theta_{r}-\psi_{r}$ is related to the band and harmonic energies by

$$
\frac{n_{r}}{2} \sin \left(\theta_{r}-\psi_{r}\right)=\frac{1}{J_{\mathrm{H}} S} \frac{d}{d \theta_{r}} \frac{E_{b}}{N}=-\frac{1}{J_{\mathrm{H}} S} \frac{d}{d \theta_{r}} \frac{E_{h}}{N},
$$

which can also be obtained by minimizing the classical energy $\mathcal{E}\left(\theta_{a}, \theta_{b}\right)=-J_{\mathrm{H}} S \sum_{i} n_{i} \cos \left(\theta_{i}-\right.$ $\left.\psi_{i}\right)+E_{h}\left(\theta_{a}, \theta_{b}\right)$ with respect to $\theta_{a}$ or $\theta_{b}$. Hence, the torque exerted by the electrons on the local moments opposes the tendency of the local moments to return to the angles that minimize $E_{h}$.

In terms of the Fermion operators $d_{\mathbf{k} \alpha}^{(r)}$ and $d_{\mathbf{k} \alpha}^{(r) \dagger}$ that diagonalize $H_{b}$, the full Hamiltonian can be expanded in a power series in $1 / \sqrt{S}$ as $H=H_{0}+H_{1}+H_{2}+\cdots$. The first-order term may be written

$$
\begin{aligned}
H_{1}= & -2 J_{\mathrm{H}} \sqrt{\frac{S}{N}} \sum_{\mathbf{k}, \mathbf{q}, r, s} d_{\mathbf{k}+\mathbf{q}, \downarrow}^{(r) \dagger} d_{\mathbf{k}, \uparrow}^{(s)}\left\{u_{\mathbf{k}+\mathbf{q}}^{(r)} u_{\mathbf{k}}^{(s)} a_{\mathbf{q}}^{(a)}+v_{\mathbf{k}+\mathbf{q}}^{(r)} v_{\mathbf{k}}^{(s)} a_{\mathbf{q}}^{(b)}\right\} \\
- & \frac{2 t}{\sqrt{S N}} \sum_{\mathbf{k}, \mathbf{q}, r, s}\left\{d_{\mathbf{k}+\mathbf{q}, \uparrow}^{(r) \dagger} d_{\mathbf{k}+\mathbf{Q}, \uparrow}^{(s)}\left(u_{\mathbf{k}+\mathbf{q}}^{(r)} x_{\mathbf{k}}^{(s)} a_{\mathbf{q}}^{(a)}+v_{\mathbf{k}+\mathbf{q}}^{(r)} y_{\mathbf{k}}^{(s)} a_{\mathbf{q}}^{(b)}\right)\right. \\
& \left.+d_{\mathbf{k}+\mathbf{q}+\mathbf{Q}, \downarrow}^{(r) \dagger} d_{\mathbf{k} \downarrow}^{(s)}\left(u_{\mathbf{k}}^{(s)} x_{\mathbf{k}+\mathbf{q}}^{(r)} a_{\mathbf{q}}^{(a)}+v_{\mathbf{k}}^{(s)} y_{\mathbf{k}+\mathbf{q}}^{(r)} a_{\mathbf{q}}^{(b)}\right)\right\}+h . c .,
\end{aligned}
$$

where

$$
\begin{gathered}
x_{\mathbf{k}}^{(a)}=u_{\mathbf{k}}^{(a)} \cos k_{y} \sin \left(\left(\theta_{a}-\theta_{b}\right) / 2\right)-u_{\mathbf{k}}^{(b)} \cos k_{x} \sin \theta_{a}, \\
y_{\mathbf{k}}^{(a)}=-u_{\mathbf{k}}^{(b)} \cos k_{y} \sin \left(\left(\theta_{a}-\theta_{b}\right) / 2\right)-u_{\mathbf{k}}^{(a)} \cos k_{x} \sin \theta_{b},
\end{gathered}
$$


and $x_{\mathbf{k}}^{(b)}=-x_{\mathbf{k}+\mathbf{Q}}^{(a)}, y_{\mathbf{k}}^{(b)}=y_{\mathbf{k}+\mathbf{Q}}^{(a)}, v_{\mathbf{k}}^{(a)}=u_{\mathbf{k}}^{(b)}$, and $v_{\mathbf{k}}^{(b)}=-u_{\mathbf{k}}^{(a)}$. The second term in Eq.(36) is produced by the torque exerted by the electrons on the local moments. Notice that $H_{1}$ is linear in the boson operators. The expectation value of the Fermion factor multiplying the boson operators in Eq.(36) vanishes provided that $\theta_{r}$ satisfy Eqs.(28) and (29). The second-order Hamiltonian can be written as

$$
H_{2}=\frac{2 J_{\mathrm{H}}}{N} \sum_{\mathbf{k}, \mathbf{q}_{1}, \mathbf{q}_{2}, r, s} \sum_{\alpha, \beta} \sigma_{\alpha \beta}^{z} d_{\mathbf{k}-\mathbf{q}_{1}, \alpha}^{(r) \dagger} d_{\mathbf{k}-\mathbf{q}_{2}, \beta}^{(s)}\left\{a_{\mathbf{q}_{1}}^{(a) \dagger} a_{\mathbf{q}_{2}}^{(a)} u_{\mathbf{k}-\mathbf{q}_{1}}^{(r)} u_{\mathbf{k}-\mathbf{q}_{2}}^{(s)}+a_{\mathbf{q}_{1}}^{(b) \dagger} a_{\mathbf{q}_{2}}^{(b)} v_{\mathbf{k}-\mathbf{q}_{1}}^{(r)} v_{\mathbf{k}-\mathbf{q}_{2}}^{(s)}\right\} .
$$

Since $J_{\mathrm{H}} / J S$ and $t / J S$ are of order $S^{0}, H_{1} / J S^{2}$ is of order $1 / \sqrt{S}$ and $H_{2} / J S^{2}$ is of order $1 / S$.

To eliminate the first-order term in $H$ and to express the Hamiltonian in terms of the true SW operators for the total spin $\mathbf{S}_{i \text {,tot }}=\mathbf{S}_{i}+\mathbf{s}_{i}$, we perform the unitary transformation 23] $H^{\prime}=e^{-U} H e^{U}$ where $U$ is constructed to satisfy [U, $\left.H_{0}\right]=H_{1}$. This transformation produces a modified second-order term $H_{2}^{\prime}=H_{2}+\left[U, H_{1}\right] / 2$. The anti-Hermitian operator $U$ that fulfills these requirements is

$$
\begin{aligned}
U= & -2 J_{\mathrm{H}} \sqrt{\frac{S}{N}} \sum_{\mathbf{k}, \mathbf{q}, r, s}\left\{d_{\mathbf{k}+\mathbf{q}, \downarrow}^{(r) \dagger} d_{\mathbf{k} \uparrow}^{(s)}\left(a_{\mathbf{q}}^{(a)} u_{\mathbf{k}+\mathbf{q}}^{(r)} u_{\mathbf{k}}^{(s)}+a_{\mathbf{q}}^{(b)} v_{\mathbf{k}+\mathbf{q}}^{(r)} v_{\mathbf{k}}^{(s)}\right) \frac{1}{\tilde{\epsilon}_{\mathbf{k}}^{(s)}-\tilde{\epsilon}_{\mathbf{k}+\mathbf{q}}^{(r)}-2 J_{\mathrm{H}} S}\right\} \\
& -\frac{2 t}{\sqrt{S N}} \sum_{\mathbf{k}, \mathbf{q}, r, s}\left\{d_{\mathbf{k}+\mathbf{q}, \uparrow}^{(r) \dagger} d_{\mathbf{k}+\mathbf{Q}, \uparrow}^{(s)}\left(a_{\mathbf{q}}^{(a)} u_{\mathbf{k}+\mathbf{q}}^{(r)} x_{\mathbf{k}}^{(s)}+a_{\mathbf{q}}^{(b)} v_{\mathbf{k}+\mathbf{q}}^{(r)} y_{\mathbf{k}}^{(s)}\right)\right. \\
& \left.+d_{\mathbf{k}+\mathbf{q}, \downarrow}^{(r) \dagger} d_{\mathbf{k}+\mathbf{Q}, \downarrow}^{(s)}\left(a_{\mathbf{q}}^{(a)} u_{\mathbf{k}+\mathbf{Q}}^{(s)} x_{\mathbf{k}+\mathbf{q}+\mathbf{Q}}^{(r)}+a_{\mathbf{q}}^{(b)} v_{\mathbf{k}+\mathbf{Q}}^{(s)} y_{\mathbf{k}+\mathbf{q}+\mathbf{Q}}^{(r)}\right)\right\} \frac{1}{\tilde{\epsilon}_{\mathbf{k}+\mathbf{Q}}^{(s)}-\tilde{\epsilon}_{\mathbf{k}+\mathbf{q}}^{(r)}}-h . c .,
\end{aligned}
$$

which is clearly of order $\left(J S^{2}\right) / \sqrt{S}$.

Performing the unitary transformation and taking the limit $J_{\mathrm{H}} S \rightarrow \infty$ produces a rather complicated expression for $H_{2}^{\prime}$. This expression can be considerably simplified by tracing over the Fermion degrees of freedom, thereby replacing the combination of Fermi operators $d_{\mathbf{k} \uparrow}^{(r) \dagger} d_{\mathbf{k}^{\prime} \uparrow}^{(s)}$ by its expectation value $\delta_{r s} \delta_{\mathbf{k}, \mathbf{k}^{\prime}} f\left(\tilde{\epsilon}_{\mathbf{k}}^{(r)}\right)$. Then $H_{2}^{\prime}$ can be written as an effective Hamiltonian for the SW operators $a_{\mathbf{q}}^{(r)}$ and $a_{\mathbf{q}}^{(r) \dagger}$ that takes the same form as the harmonic Hamiltonian of Eq.(6) but with revised coefficients $\tilde{A}_{\mathbf{k}}^{(r, s)}=A_{\mathbf{k}}^{(r, s)}+C_{\mathbf{k}}^{(r, s)}+D_{\mathbf{k}}^{(r, s)}$ and $\tilde{B}_{\mathbf{k}}^{(r, s)}=$ $B_{\mathbf{k}}^{(r, s)}+E_{\mathbf{k}}^{(r, s)}$. The new contributions to these coefficients are

$$
\begin{aligned}
C_{\mathbf{k}}^{(r, s)}= & -\frac{1}{N} \sum_{\mathbf{q}, l} f\left(\tilde{\epsilon}_{\mathbf{q}}^{(l)}\right)\left\{u_{\mathbf{q}}^{(l) 2}\left(2 t^{\prime} \cos \theta_{a} \cos \left(k_{x}+q_{x}\right)+\tilde{\epsilon}_{\mathbf{q}}^{(l)}\right) \delta_{r, a} \delta_{s, a}\right. \\
& +v_{\mathbf{q}}^{(l) 2}\left(2 t^{\prime} \cos \theta_{b} \cos \left(k_{x}+q_{x}\right)+\tilde{\epsilon}_{\mathbf{q}}^{(l)}\right) \delta_{r, b} \delta_{s, b} \\
& \left.+2 t^{\prime} \cos \left(\left(\theta_{a}-\theta_{b}\right) / 2\right) \cos \left(k_{y}+q_{y}\right) u_{\mathbf{q}}^{(l)} v_{\mathbf{q}}^{(l)}\left(\delta_{r, a} \delta_{s, b}+\delta_{r, b} \delta_{s, a}\right)\right\}
\end{aligned}
$$




$$
\begin{aligned}
D_{\mathbf{k}}^{(r, s)}= & \frac{4 t^{\prime 2} J S^{2}}{N} \sum_{\mathbf{q}, l, m}\left(u_{\mathbf{k}+\mathbf{q}}^{(l)} x_{\mathbf{q}}^{(m)} \delta_{r, a}+v_{\mathbf{k}+\mathbf{q}}^{(l)} y_{\mathbf{q}}^{(m)} \delta_{r, b}\right) \\
& \left(u_{\mathbf{k}+\mathbf{q}}^{(l)} x_{\mathbf{q}}^{(m)} \delta_{s, a}+v_{\mathbf{k}+\mathbf{q}}^{(l)} y_{\mathbf{q}}^{(m)} \delta_{s, b}\right) \frac{f\left(\tilde{\epsilon}_{\mathbf{q}+\mathbf{Q}}^{(m)}\right)-f\left(\tilde{\epsilon}_{\mathbf{k}+\mathbf{q}}^{(l)}\right)}{\tilde{\epsilon}_{\mathbf{q}+\mathbf{Q}}^{(m)}-\tilde{\epsilon}_{\mathbf{k}+\mathbf{q}}^{(l)}}, \\
E_{\mathbf{k}}^{(r, s)}= & \frac{2 t^{\prime 2} J S^{2}}{N} \sum_{\mathbf{q}, l, m}\left(u_{\mathbf{q}+\mathbf{Q}}^{(m)} x_{\mathbf{k}+\mathbf{q}+\mathbf{Q}}^{(l)} \delta_{r, a}+v_{\mathbf{q}+\mathbf{Q}}^{(m)} y_{\mathbf{k}+\mathbf{q}+\mathbf{Q}}^{(l)} \delta_{r, b}\right) \\
& \left(u_{\mathbf{k}+\mathbf{q}}^{(l)} x_{\mathbf{q}}^{(m)} \delta_{s, a}+v_{\mathbf{k}+\mathbf{q}}^{(l)} y_{\mathbf{q}}^{(m)} \delta_{s, b}\right) \frac{f\left(\tilde{\epsilon}_{\mathbf{q}+\mathbf{Q}}^{(m)}\right)-f\left(\tilde{\epsilon}_{\mathbf{k}+\mathbf{q}}^{(l)}\right)}{\tilde{\epsilon}_{\mathbf{q}+\mathbf{Q}}^{(m)}-\tilde{\epsilon}_{\mathbf{k}+\mathbf{q}}^{(l)}} .
\end{aligned}
$$

Spin excitation frequencies $\nu_{\mathbf{q}}^{ \pm}$are given by Eq.(13) with $\tilde{A}_{\mathbf{k}}^{(r, s)}$ and $\tilde{B}_{\mathbf{k}}^{(r, s)}$ replacing the harmonic coefficients. Note that the torque terms in $H_{1}$ produce $D_{\mathbf{q}}^{(r, s)}$ and $E_{\mathbf{q}}^{(r, s)}$, both of which vanish in the FM phase. In the CAF phase, these terms are required to preserve rotational symmetry and the relations $\omega_{\mathbf{Q}}=0$ and $\omega_{0}=B$.

An analytic expression for the SW frequency is possible in the FM phase:

$$
\begin{aligned}
\omega_{\mathbf{k}}= & B+J S\left(3-\eta+(\eta-1) \cos k_{x}\right)+K\left(2-\cos k_{x}\right) / 4 S \\
& -J S \sqrt{(1+\eta)^{2}\left(1-\cos k_{x}\right)^{2}+\left(2+K / 4 J S^{2}\right)^{2} \cos ^{2} k_{y}},
\end{aligned}
$$

which generalizes Eq.(15) for the Villain model. The FM phase becomes unstable when $\omega_{\mathbf{Q}}=0$, which yields the same condition for the CAF-FM phase boundary as Eq.(30). In the FM phase, the SW stiffnesses are the sum of DE and Heisenberg contributions: $D_{\mathrm{sw}}^{x}=K / 8 S+J S(1-\eta) / 2$ and $D_{\mathrm{sw}}^{y}=K / 8 S+J S$, both independent of field. For $J=0$, these results agree with the SW frequencies of the DE model first obtained by Furukawa 35]. When $\eta=-1$, the SW's are isotropic because all nearest-neighbor interactions equal $J$.

In the CAF phase, the SW frequency and stiffnesses are solved numerically by integrating over the first Brillouin zone to obtain $C_{\mathbf{k}}^{(r, s)}, D_{\mathbf{k}}^{(r, s)}$, and $E_{\mathbf{k}}^{(r, s)}$. Results for the SW frequency are plotted in Fig.11 for $\eta=3, B^{\prime}=0$, and several values of $t^{\prime}$. For all $t^{\prime} \leq t_{c}^{\prime} \approx 21.2$, the SW frequency vanishes at both the FM and AFM Bragg vectors. Above the phase separation region around $t^{\prime} \approx 10.0$ but below $t_{c}^{\prime}$, $\omega_{\mathrm{k}}$ develops kinks that correspond to transitions across the neck of the $a$ FS $\left(\Delta \mathbf{q}_{1}=\mathbf{Q}-\mathbf{k}_{1} \approx 0.14 \pi \hat{\mathbf{x}}\right.$ for $\left.t^{\prime}=10.2\right)$ and across the length of the $b$ FS $\left(\Delta \mathbf{q}_{2}=\mathbf{Q}-\mathbf{k}_{2} \approx 0.33 \pi \hat{\mathbf{x}}\right.$ for $\left.t^{\prime}=10.2\right)$. These transitions are sketched in Fig.10. Since the nesting across $\Delta \mathbf{q}_{2}$ is much weaker than across $\Delta \mathbf{q}_{1}$, the kink in the SW frequency at 


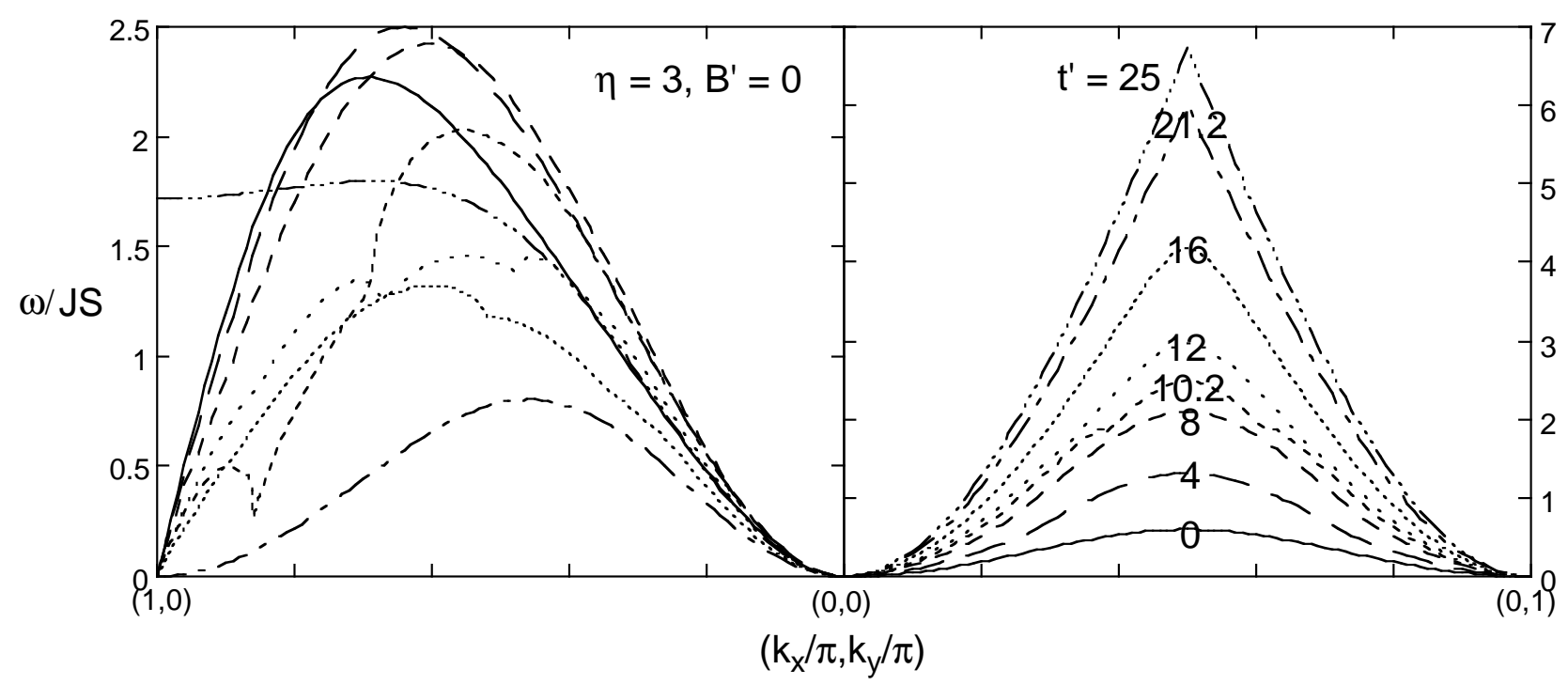

FIG. 11: The SW frequencies for $p=0.66, \eta=3, B^{\prime}=0$, and various values of $t^{\prime}$. Different frequency scales are used on either side of $\mathbf{k}=0$.

$\mathbf{k}_{2}$ is much weaker than the one at $\mathbf{k}_{1}$. As $t^{\prime} \rightarrow 10.0, \Delta \mathbf{q}_{i} \rightarrow 0$ so the kinks at $\mathbf{k}_{1}$ and $\mathbf{k}_{2}$ merge with $\mathbf{Q}$. Whereas $\omega_{\mathbf{k}}$ is a non-monotonic function of $t^{\prime}$ along the $x$ direction, it is a monotonically increasing function of $t^{\prime}$ along the $y$ direction.

As plotted in Fig.6(b) for $\eta=2$, the SW stiffness in the $x$ direction reaches a minimum at $t_{c}^{\prime} \approx 13.2$, above which both $D_{\mathrm{sw}}^{x}$ and $D_{\mathrm{sw}}^{y}$ are linearly increasing functions of $t^{\prime}$. The stiffnesses in the $x$ and $y$ directions cross in the region of phase separation, where the SW's become isotropic. Notice that $D_{\mathrm{sw}}^{a v}$ increases by about $65 \%$ as $t^{\prime}$ increases from zero to $t_{c}^{\prime}$. For $\eta=3, D_{\mathrm{sw}}^{a v}$ doubles in this interval [27]

With increasing $\eta$, the SW stiffnesses plotted in Fig.7(b) for $t^{\prime}=10$ and $B=0$ again cross in the region of phase separation around $\eta=3.0$. For larger $\eta, D_{\mathrm{sw}}^{x}>D_{\mathrm{sw}}^{y}$. Due to the strong increase in $D_{\mathrm{sw}}^{x}$ with $\eta$, the average SW stiffness actually grows as the local moments become more non-collinear. Similar behavior was found when $t=0$ in Fig.2, where $D_{\mathrm{sw}}^{a v}$ was also found to be an increasing function of $\eta$.

The effect of a magnetic field is shown in Figs.8(b) and 12 for $\eta=2$ and $t^{\prime}=3$. Absent from Fig.8(b) is the region of very low fields, where the behavior of the SW stiffness is complicated by the dependence of the function $D_{\mathrm{sw}}^{\alpha}\left(k_{\alpha}, B\right)=\left(\omega_{\mathbf{k}}-B\right) / k_{\alpha}^{2}$ on the ratio $k_{\alpha} / \sqrt{B^{\prime}}$ 29]. For larger fields, there is a gradual increase in $D_{\mathrm{sw}}^{y}$ and a decrease in $D_{\mathrm{sw}}^{x}$ as $B$ grows from 0 to $B_{c} \approx 4.8 J S$. The drop in $D_{\mathrm{sw}}^{x}$ with field is caused by the growth of the 


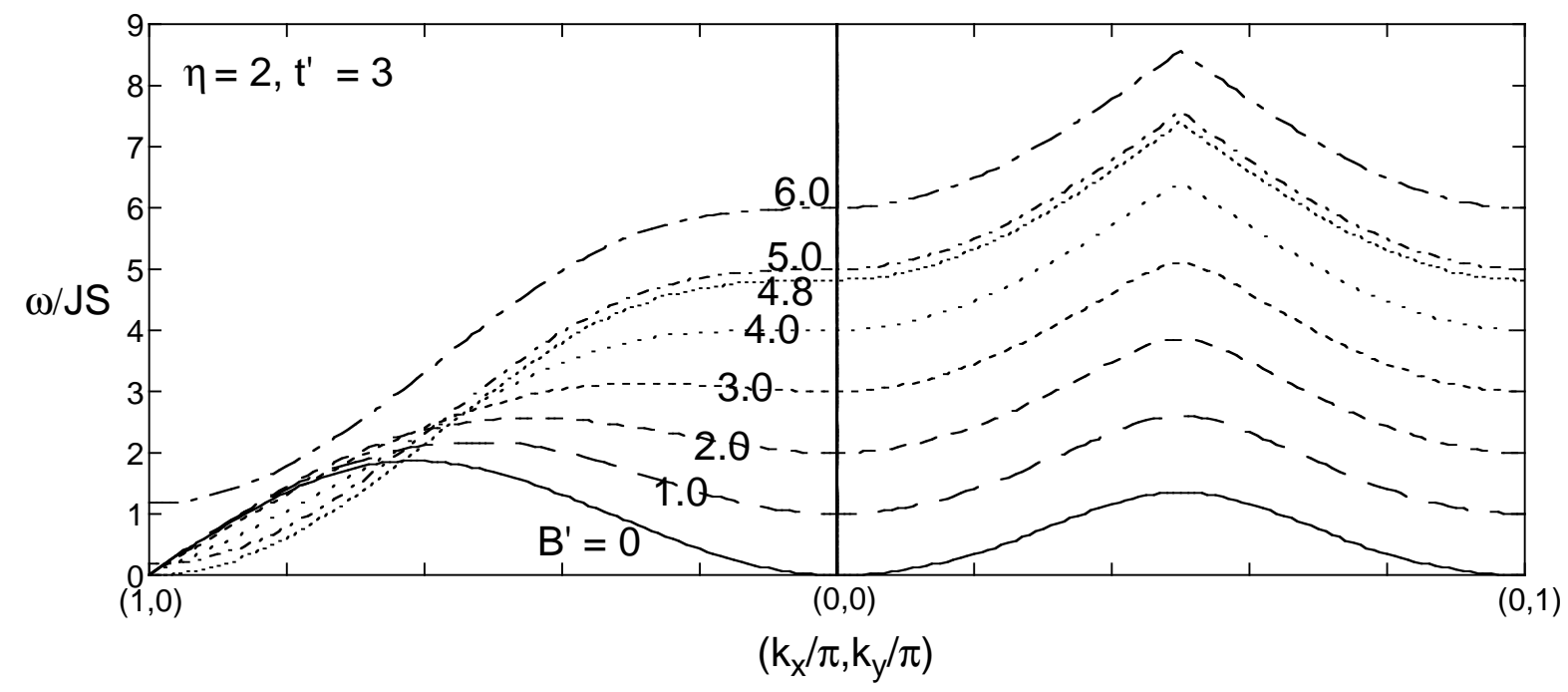

FIG. 12: The SW frequencies for $p=0.66, \eta=2, t^{\prime}=3$, and various values of $B^{\prime}$.

SW gap $\omega_{0}=B$ at $\mathbf{k}=0$ while the SW frequency continues to vanish at $\mathbf{Q}=(\pi, 0)$ for $B<B_{c}$, as seen in Fig.12. Hence, the average SW stiffness drops from roughly 1.2JS at $B=0$ to $0.54 J S$ at $B_{c}$. Because a magnetic field very quickly destroys the region of phase separation, the crossing point of the $x$ and $y$ stiffnesses does not coincide with a region of phase separation. As expected, the SW stiffnesses are independent of field above $B_{c}$. Notice from Figs.11 and 12 that the SW velocity at $\mathbf{Q}$ softens within the CAF phase as $t$ approaches $t_{c}$ or $B$ approaches $B_{c}$. This behavior is quite general and occurs at the CAF-FM phase boundary of any magnetic system [31] .

\section{DISCUSSION AND CONCLUSION}

Applying the results of this work to the manganites requires an estimation of the relevant parameters. Using $S=3 / 2, \eta J S \approx 8.6 \mathrm{meV}, \eta \sim 1$ [36], and $t \approx 200 \mathrm{meV}$ for the metallic phase gives $t^{\prime} \approx 32$ and $B^{\prime} \approx 0.028 B(T)$. When $\eta=1$, the critical values of the DEV model are $t_{c}^{\prime}=5.3$ in zero field and $B_{c}^{\prime}=2.5$ for $t^{\prime}=0$. Hence, the metallic value for the hopping energy is more than sufficient to stabilize the FM phase but an applied field of $3 \mathrm{~T}$ cannot align the local moments when $t^{\prime}$ is much below $t_{c}^{\prime}$.

Metal-insulator transitions in many of the manganites have been successfully interpreted within the framework of percolation theory [37, 38]. When the FM fraction $f$ exceeds a critical value $f_{c}$, a FM backbone spans the sample and the system becomes metallic. 
The percolation threshold $f_{c}$ in those manganites lies between 0.17 and 0.19 . But it is rather difficult to explain the jump in the SW stiffness observed in $\operatorname{Pr}_{0.7} \mathrm{Ca}_{0.3} \mathrm{MnO}_{3}$ [1] within the framework of percolation theory. Assuming that the metal-insulator transition in $\operatorname{Pr}_{1-x} \mathrm{Ca}_{x} \mathrm{MnO}_{3}$ is produced by the percolation of metallic FM clusters [10], there is no reason why the SW stiffness should change when a FM backbone spans the sample. Within percolation theory, one would more naturally expect the SW stiffness to gradually increase with field as a growing fraction of the sample becomes metallic.

Estimates of the FM fraction $f$ in $\operatorname{Pr}_{1-x} \mathrm{Ca}_{x} \mathrm{MnO}_{3}$ manganites also pose a difficulty for percolation theory. The accepted range for the percolation threshold $f_{c}$ within continuum models falls between 0.15 and 0.22 . While one experimental estimate of $f_{c} \approx 0.08$ [10] at the metal-insulator transition lies well below this accepted range, a recent estimate of $f_{c} \approx 0.6$ 13. based on more complete magnetization data lies well above this range. At zero field and low temperatures, estimates for $f$ range from 0.3 [13] to 0.5 [9]. If those estimates are correct and the FM regions are metallic, then the percolation threshold would already be exceeded and the low-temperature phase would be metallic in zero field.

On the other hand, our results clearly indicate that the jump in the SW stiffness at a field of $3 \mathrm{~T}$ cannot be produced by simply aligning the AFM regions while keeping the bandwidth $\sim t$ fixed. As found in Fig.8(b), a magnetic field would act to suppress rather than enhance the average SW stiffness under those conditions. Moreover, a field of $3 \mathrm{~T}$ corresponding to $B^{\prime} \approx 0.086 \ll B_{c}^{\prime}$ will have little affect on the alignment of the local moments. Whereas experiments indicate that the AFM regions begin to shrink under a magnetic field greater than $3 \mathrm{~T}$, a sizeable fraction of the sample remains AFM even above the metal-insulator transition [10, 11, 13].

The large increase in the SW stiffness predicted by our work as $t^{\prime}$ increases from 0 to $t_{c}^{\prime}$ suggests an entirely different scenario: at the critical field, electrons in the insulating and possibly canted FM regions delocalize as the hopping energy $t$ sharply increases. Since the integrated optical weight is just $K \sim t$, the jump in the hopping energy at $3 \mathrm{~T}$ should be observable in optical measurements. Indeed, measurements by Okimoto et al. [7] on $\operatorname{Pr}_{0.6} \mathrm{Ca}_{0.4} \mathrm{MnO}_{3}$ reveal a rapid rise in $\sigma(\omega)$ and a rapid drop in the CDW gap near the critical field. If the percolation threshold for the FM regions is exceeded when the electrons delocalize, as suggested by the measurements discussed above, then the jump in the SW stiffness will coincide with the metal-insulator transition. Otherwise, the metal-insulator 
transition will occur at a slightly larger field.

Supporting this picture are the plethora of probes that can produce a metal-insulator transition in $\operatorname{Pr}_{1-x} \mathrm{Ca}_{x} \mathrm{MnO}_{3}(0.3 \leq x \leq 0.4)$. Besides a magnetic field, application of an electric field 39], high pressure [40], exposure to x-rays [41], and exposure to visible light [4] all induce a metal-insulator transition. The identical resistivities produced by application of a magnetic field or exposure to x-rays [41] suggest a common mechanism: the excitation of charge carriers out of polaronic traps produced by the electron-lattice coupling. The subsequent relaxation of the lattice [9, 41] may prevent retrapping of the electrons.

The jump in the SW stiffness as a function of field in $\operatorname{Pr}_{0.7} \mathrm{Ca}_{0.3} \mathrm{MnO}_{3}$ [1] also bares a striking resemblance to the jump in the SW stiffness observed in $\mathrm{La}_{1-x} \mathrm{Ca}_{x} \mathrm{MnO}_{3}$ at the metal-insulator transition with $x \approx 0.22$ [43]. In fact, the sizes of the SW stiffnesses on either side of the doping-induced transition in $\mathrm{La}_{1-x} \mathrm{Ca}_{x} \mathrm{MnO}_{3}$ are almost exactly the same as on either side of the field-induced transition in $\operatorname{Pr}_{0.7} \mathrm{Ca}_{0.3} \mathrm{MnO}_{3}$. This suggests that there are also large increases in the hopping energy and electronic kinetic energy in $\mathrm{La}_{1-x} \mathrm{Ca}_{x} \mathrm{MnO}_{3}$ at the critical concentration of $x \approx 0.22$.

Compare the jump in the SW stiffness across the metal-insulator transition with the smooth dependence of the spin-diffusion coefficient $\Lambda$ [4, 43], which gives the lifetime $\tau(\mathbf{k})=$ $1 / \Lambda k^{2}$ of paramagnetic spin fluctuations. Measurements [43] of the spin-diffusion coefficient were performed just below $T_{\mathrm{C}}$, where paramagnetic spin relaxation occurs within polaronic regions of the FM phase. While $D_{\mathrm{sw}}$ is a linear function of the electronic kinetic energy in the FM phase, $\Lambda \chi$ depends only on doping and is independent of the electronic bandwidth $W$ in the low-temperature limit $T \ll W[4]$. So long as the bandwidth of the polaronic regions remains large compared to the temperature, the spin-diffusion coefficient will not change across the metal-insulator transition.

Except in a very narrow range of parameters, phase separation is absent in the DEV model. Phase separation appears quite commonly in DE models with AFM Heisenberg interactions 1] and even occurs near $p=1$ in a DE model without Heisenberg interactions 44]. Results for the DEV model contrast with both a DE model with AFM interactions between all neighboring local moments [20, 21, 22, 23] and a DE model with FM interactions in plane but AFM interactions between neighboring planes [45]. Due to the high symmetry of both models, AFM order is not frustrated when $t=0$. But in both cases, phase separation occurs before the AFM interactions become strong enough to cant the spins. Work by 
Golosov [21, 23] indicates that the canted phase is destabilized by the presence of local degeneracies 24] that are absent in the DEV model. Our results suggest that phase separation only occurs within a very narrow range of parameters when the AFM Heisenberg interactions are frustrated and local degeneracies are absent. For doping concentrations away from multiples of a quarter filling, long-range orbital ordering is impossible and magnetic frustration may be present in a wide range of manganites. Therefore, the DE model may not provide as straightforward a pathway to phase separation in the manganites as believed. A much more important role may be played by the quenched disorder associated with chemical inhomogeneities [46].

Román and Soto [31] pointed out that the nature of the FM and AFM regions can be probed by measuring the SW spectrum about the AFM Bragg vector $\mathbf{Q}$. In a CAF, the SW's are not gapped for any field $B$ below $B_{c}$, as shown in Figs.4 and 12. By contrast, the SW's of a collinear AFM contain a gap at $\mathbf{Q}$ that grows linearly with field. So measurements of the SW spectrum about $\mathbf{Q}$ may be used to determine whether local regions are canted. As discussed elsewhere [29] and seen in the inset to Fig.3(b), the rapid increase in the SW stiffness for fields near $B^{\star}$ is another signature of a canted phase. The absence of this behavior in $\operatorname{Pr}_{1-x} \mathrm{Ca}_{x} \mathrm{MnO}_{3}$ for $0.3 \leq x \leq 0.4$ strongly suggests that the local AFM regions are not canted.

Since CE-type AFM ordering occurs at half-filling, the local charge ordering observed in the manganites $\mathrm{Pr}_{0.6} \mathrm{Ca}_{0.4} \mathrm{MnO}_{3}$ [7, 32] and $\mathrm{La}_{0.7} \mathrm{Ca}_{0.3} \mathrm{MnO}_{3}$ [2, 3] would be simplified if the polaronic regions were rich in holes and poor in electrons compared to the bulk. Our model provides a natural explanation for this behavior, since the electronic fraction on $b$ sites is substantially smaller than the fraction on $a$ sites as the electrons avoid regions with more pronounced AFM order.

This paper has studied the general effects of AFM interactions and non-collinearity on the magnetic ordering and spin dynamics of DE systems. The competition between DE and AFM interactions is responsible for several interesting properties. In a narrow region of hopping energies, weak phase separation occurs as the FS topology changes from closed to open. Because electrons prefer to occupy sites that are coupled by FM interactions, a CDW appears in the absence of a CDW gap. For finite Hund's coupling, the electron spins are more closely aligned than the local moments of the CAF. The CAF becomes unstable above critical values of field and hopping energy and below a critical value of $\eta$. Perhaps 
the most surprising result is that DE changes none of the qualitative features of the CAF state. The SW gap $\omega_{0}$ at $\mathbf{k}=0$ remains equal to $B$ and the gap $\omega_{\mathbf{Q}}$ at the AFM Bragg vector $\mathbf{Q}$ continues to vanish for any hopping energy below $t_{c}$. The average $\mathrm{SW}$ stiffness still softens with magnetic field and hardens with strengthening AFM interactions; the SW velocity at $\mathbf{Q}$ still vanishes at the CAF-FM transition. Clearly, a great deal can be learned about the general properties of CAF's, even of the more complex variations that appear in many manganites, by considering simple models such as the DEV model.

It is a pleasure to acknowledge helpful conversations with Drs A Chernyshev, P-C Dai, E Dagotto, J Fernandez-Baca, D Golosov, M Katsnelson, N Furukawa, W Saslow, and A Zheludev. This research was sponsored by the US Department of Energy under contract DE-AC05-00OR22725 with Oak Ridge National Laboratory, managed by UT-Battelle, LLC.

[1] For an overview of phase separation in the manganites, see Moreo A, Yunoki S, and Dagotto E 1999 Science 2832034 and Dagotto E, Hotta T, and Moreo A 2001 Phys. Rep. 2331.

[2] Adams C P, Lynn J W, Mukovskii Y M, Arsenov A A, and Shulyatev D 2000 Phys. Rev. Lett. 85, 3954 .

[3] Koo T Y, Kiryukhin B, Sharma P A, Hill J P, and Cheong S-W 2001 Phys. Rev. B 64220405.

[4] Chernyshev A and Fishman R S 2003 Phys. Rev. Lett. 90177202.

[5] Jirak Z, Krupicka S, Simsa Z, Dlouha M, and Vratislav S, 1985 J. Magn. Magn. Mater. 53 153.

[6] Yoshizawa H, Kawano H, Tomioka Y, and Tokura Y 1996 Phys. Rev. B 52 R13145; 1996 J. Phys. Soc. Japan 651043.

[7] Okimoto Y, Tomioka Y, Onose Y, Otsuka Y, and Tokura Y 1999 Phys. Rev. B 597401.

[8] Deac I G, Mitchell J F, and Schiffer P 2001 Phys. Rev. B 63172408.

[9] Radaelli P G, Ibberson R M, Argyriou D N, Casalta H, Anderson K H, Cheong S-W, and Mitchell J F 2001 Phys. Rev. B 63172419.

[10] Hardy V, Wahl A, and Martin C 2001 Phys. Rev. B 64064402.

[11] Fernandez-Baca J A, Dai P-C, Kawano-Furukawa H, Yoshizawa H, Plummer E W, Katano S, Tomioka Y, and Tokura Y 2002 Phys. Rev. B 66054434.

[12] Simon Ch, Mercone S, Guiblin N, Martin C, Brûlet A, and André G 2002 Phys. Rev. Lett. 89 
207202.

[13] Mercone S, Hardy V, Martin C, Simon C, Saurel D, and Brûlet A 2003 Phys. Rev. B 68 094422.

[14] Anderson P W and Hasegawa H 1995 Phys. Rev. 100675.

[15] DeGennes P-G 1960 Phys. Rev. 118141.

[16] Villain J 1977 J. Phys. C 101717.

[17] Berge B, Diep H T, Ghazali A, and Lallemand P 1986 Phys. Rev. B 343177.

[18] Gabay M, Garel T, Parker G N, and Saslow W M 1989 Phys. Rev. B 40264.

[19] Saslow W M and Erwin R 1992 Phys. Rev. B 454759.

[20] Yunoki S and Moreo A 1998 Phys. Rev. B 586403.

[21] Golosov D I, Norman M R, and Levin K 1998 Phys. Rev. B 588617.

[22] Kagan M Yu, Khomskii D I, and Mostovoy M V 1999 Eur. Phys. J. B 12217.

[23] Golosov D I 2000 Phys. Rev. Lett. 84 3974; 2000 J. Appl. Phys. 87 5804; 2002 ibid. 917508.

[24] In the canted phase of a two-sublattice model with AFM interactions, the local moment on any site of sublattice $a$ must make an angle $\Theta$ with the local moments on sublattice $b$. So the possible orientations for any a spin trace out a cone around the equilibrium direction of the $b$ spins $[21,23]$. In the canted phase of a two-sublattice model with FM interactions in plane but AFM interactions between planes, the moments in any plane $a$ are free to rotate about the equilibrium direction of the moments on neighboring planes $b$. These local or planar degeneracies are absent in the DEV model.

[25] Mizokawa T, Khomskii D I, and Sawatzky G A 2000 Phys. Rev. B 63024403.

[26] While the FM coupling between neighboring $\mathrm{Mn}^{4+}$ and $\mathrm{Mn}^{3+}$ ions are induced by the hopping of electrons those sites, that coupling is significantly enhanced by the polaronic distortions of the $\mathrm{O}$ atoms around some $\mathrm{Mn}^{4+}$ ions [25]. So the Heisenberg coupling $J$ incorporates the enhancement of the FM interaction beyond that produced by the uniform hopping of electrons between neighboring sites on an undistorted lattice.

[27] Fishman R S 2004 Phys. Rev. B (in press).

[28] Walker L R and Walstedt R E 1980 Phys. Rev. B 223816.

[29] Fishman R S (unpublished).

[30] Since there are four inequivalent sites on the Villain lattice, it might seem that the first Brillouin zone should be reduced in both the $x$ and $y$ directions. However, all $a$ or $b$ sites experience 
the same environment regardless of whether the spins tilt to the $+x$ or $-x$ directions: each $a$ spin, for example, makes an angle of $\theta_{b}-\theta_{a}$ with its $b$ neighbors and an angle $2 \theta_{a}$ with its $a$ neighbors.

[31] Román J M and Soto J 2000 Phys. Rev. B 623300.

[32] Asaka T, Yamada S, Tsutsumi S, Tsurata C, Kimoto K, Arima T, and Matsui Y 2002 Phys. Rev. Lett. 88097201.

[33] Halboth C J and Metzner W 2000 Phys. Rev. Lett. 855162.

[34] Valenzuela B and Volzmediano M A H 2001 Phys. Rev. B 63153103.

[35] Furukawa N 1995 J. Phys. Soc. Japan 64 2754; 1999 Physics of Manganites ed T A Kaplan and S D Mahanti (New York: Plenum) p 1.

[36] Perring T G, Aeppli G, Moritomo Y, and Tokura Y 1997 Phys. Rev. Lett. 783197.

[37] Kim K H, Uehara M, Hess C, Sharma P A, and Cheong S-W 2000 Phys. Rev. Lett. 842961.

[38] Babushkina N A, Taldenkov A N, Belova L M, Chistotina E A, Gorbenko O Yu, Kaul A R, Kugel K I, and Khomskii D I 2000 Phys. Rev. B 62 R6081.

[39] Asamitsu A, Tomioka Y, Kuwahara H, and Tokura Y 1997 Nature 38850.

[40] Moritomo Y, Kuwahara H, Tomioka Y, and Tokura Y 1997 Phys. Rev. B 557540.

[41] Kiryukhin V, Casa D, Hill J P, Keimer B, Vigliante A, Tomioka Y, and Tokura Y 1997 Nature 386813.

[42] Miyano K, Tanaka T, Tomioka Y, and Tokura Y 1997 Phys. Rev. Lett. 784257.

[43] Dai P-C, Fernandez-Baca J A, Plummer E W, Tomioka Y, and Tokura Y 2001 Phys. Rev. B 64224429.

[44] Yunoki S, Hu J, Malvezzi A L, Moreo A, Furukawa N, and Dagotto E 1998 Phys. Rev. Lett. 80845.

[45] Arovas D P and Guinea F 1998 Phys. Rev. B 589150.

[46] Burgy J, Mayr M, Martin-Mayor V, Moreo A, and Dagotto E 2001 Phys. Rev. Lett. 87277202. 